\title{
G-RMSD: Root Mean Square Deviation Based Method for Three-dimensional Molecular Similarity Determination
}

\author{
Tomonori Fukutani*, Kohei Miyazawa*, Satoru Iwatał Hiroko Satoh ${ }^{\ddagger \S}$
}

March 4, 2020

\begin{abstract}
We present the Generalized Root Mean Square Deviation (G-RMSD) method. GRMSD is an optimization method to calculate the minimal RMSD value of two atomic structures by optimal superimposition. The method is not restricted to systems with an equal number of atoms or a unique atom matching and can handle any type of chemical structure, including transition states and non-valence bond structures. It requires only Cartesian coordinates for the structures, but can also include further information, i.e. atom and bond types. Applications of G-RMSD to the classification of $\alpha$-D-glucose conformers and 3D partial structure search using a dataset containing equilibrium (EQ), dissociation channel (DC), and transition state (TS) structures are demonstrated. We find that G-RMSD allows for a successful classification and mapping for a wide variety of molecular structures.
\end{abstract}

Keywords: $\quad$ Optimal superimposition for minimum RMSD, 3D-Molecular similarity, 3D-Database search, Transition states, Non-valence structures

*Department of Mathematical Informatics, The University of Tokyo, Hongo 7-3-1, Bunkyo, 113-8654 Tokyo, Japan

${ }^{\dagger}$ Department of Mathematical Informatics, The University of Tokyo, Hongo 7-3-1, Bunkyo, 113-8654 Tokyo, Japan. Corresponding Author. E-mail:iwata@mist.i.u-tokyo.ac.jp

${ }^{\ddagger}$ Department of Chemistry, University of Zurich, Winterthurerstrasse 190, 8056 Zurich. Corresponding Author. E-mail: hiroko.satoh@chem.uzh.ch

${ }^{\S}$ Research Organization of Information and Systems (ROIS), Toranomon 4-3-13, Minato, 105-0001 Tokyo, Japan. 


\section{INTRODUCTION}

The measure of molecular similarity is a primary issue for processing databases of chemical structures. The chemical data processing is usually conducted on the basis of two-dimensional (2D) chemical structures. The 2D-based methods make it possible to process large data with low computational cost by handling chemical structures as topological graphs. Chemoinformatics has contributed to provide various useful mathematical and chemical methods for the measures of 2D-molecular similarity since 1960s, which have been until now playing a central role for chemical data processing. ${ }^{1-4}$ For example, 2D-based methods are used in most of the major chemical databases, including the CAS Registry from Chemical Abstract Service. ${ }^{5}$ The exploration of chemical space, intensively studied since 1990s especially for drug design, has been primarily conducted in the 2 D-basis. ${ }^{6-9}$

Another measure of molecular similarity is needed for three-dimensional (3D)-chemical

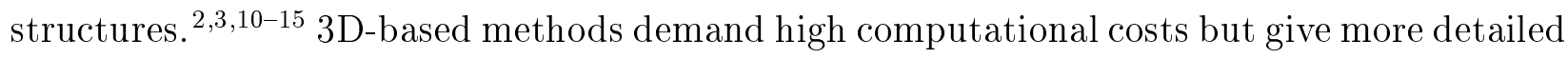
information on chemical structures, which are often strongly related to their properties, such as biological activity and chemical reactivity. The number of 3D-chemical structure databases is still smaller than that of $2 \mathrm{D}$-ones but has been recently increasing. The Protein Data Bank (PDB) ${ }^{16,17}$ and the Cambridge Structural Database (CSD) ${ }^{18,19}$ are longestablished databases of 3D-chemical structures. Their contents are experimental structures mostly determined by X-ray analysis.

New databases of calculated 3D-structures have appeared and been getting into the

mainstream. ${ }^{20-30}$ They contain structures and energies calculated with different types of methods, including high level quantum mechanical (QM) calculations. Most of the QM-based 3D structural databases (QM-databases) consist of only equilibrium (EQ) structures, but some databases contain also transition state (TS) structures. For example, RMap database, recently reported, ${ }^{27-29}$ contains reaction route maps (r-maps) composed of EQ structures and dissociation channels (DC) connected by intrinsic reaction coordinates (IRC) via TS structures. Constant increase of computer performance and rapidly growing interests of data-driven molecular discovery using machine learning (ML) and artificial intelligence (AI) technologies have accelerated the development of QM-databases. 
Those new QM-databases require new methods to handle more diverse types of molecular structures, such as structures beyond valence bond theory, TS structures, transformations between EQ and TS structures along a reaction coordinate, and structures in excited states. Furthermore, some of those types of structure might be able to be captured also in experiments by advanced measurement technologies, such as operando spectroscopy. These structures cannot be treated with the methods developed for the traditional structural databases, which generally cover only valence structures. There are useful measures of 3D-molecular similarity available. ${ }^{2,3,10-15}$ However, the trend of the QM-databases and the measurement technology innovations necessitate more general methods that can handle diverse molecular structures. It is known that such advanced 3D similarity measures are essential for ML applications. ${ }^{31}$

A method that can handle such structures beyond valence bond theory is the root mean square deviation (RMSD) method. It is commonly used for comparing 3D-molecules, such as for the analysis of trajectories obtained from molecular dynamics (MD) simulations and measuring bio-molecules. ${ }^{32-39}$ However, the RMSD method can only be applied for comparing molecules of the same size and requires a unique atom mapping or a fixed superimposition between the molecules. These restrictions need to be removed for a general use in 3Dchemical data processing.

We have tackled this problem and developed a new method to determine 3D-molecular similarity called Generalized-RMSD (G-RMSD) for the purpose of handling general 3Dchemical information. G-RMSD is an optimization method to get an optimal superimposition for minimum RMSD without a unique atom mapping. It solves the problem of comparing molecules of different size without a unique atom mapping between the molecules. A fixed superimposition is not necessary either. The method can be applied to various types of molecules, including intermediates and non-valence structures. We describe the detailed algorithm of G-RMSD together with results of test calculations using model numerical datasets. We then demonstrate the applications to pyranose ring conformer classification and 3D-partial structure search using datasets containing EQ, DC, and TS structures obtained by QM-based global reaction pathway explorations. 


\section{COMPUTATIONAL METHODOLOGY}

We describe the mathematical details of the G-RMSD method in this section.

\subsection{Mathematical basis of RMSD method}

If two molecules $A$ and $B$ composed of $n$ atoms are compared, the datasets are described as $A=\left\{a_{1}, a_{2}, \ldots, a_{n}\right\}$ and $B=\left\{b_{1}, b_{2}, \ldots, b_{n}\right\}$, where $a_{i}$ and $b_{i}(i=1,2 \ldots n)$ are vectors that correspond to the $3 \mathrm{D}$ coordinates of the $i$ th atoms of $A$ and $B$, respectively. In other words, $A$ and $B$ are $3 \times n$ matrices. Then, the RMSD value with respect to an orthogonal matrix $R$ (rotation) and a translation vector $v$ is defined by

$$
\operatorname{RMSD}(A, B, R, v):=\sqrt{\frac{1}{n} \sum_{i=1}^{n}\left\|a_{i}-R b_{i}-v\right\|^{2}} .
$$

The similarity indicator $\mu(A, B)$ between molecules $A$ and $B$ is obtained by minimizing the $\operatorname{RMSD}(A, B, R, v)$ value against $R$ and $v$, i.e.,

$$
\mu(A, B):=\min _{R, v} \operatorname{RMSD}(A, B, R, v)
$$

The similarity indicator $\mu$ can be computed efficiently by using singular value decomposition. ${ }^{40}$

\subsection{Development of G-RMSD method}

The RMSD method requires a unique atom mapping between $A$ and $B$, and usually the mapping is sequentially done, meaning, $a_{i}$ is compared with $b_{i}$. Therefore, for the case without any specification of such atom mapping, the definition needs to be extended by a permutation $\sigma$ on $\{1, \ldots, n\}$ :

$$
\operatorname{RMSD}(A, B, R, v, \sigma):=\sqrt{\frac{1}{n} \sum_{i=1}^{n}\left\|a_{i}-R b_{\sigma(i)}-v\right\|^{2}}
$$

Then, the similarity indicator $\mu(A, B)$ is obtained by minimizing the $\operatorname{RMSD}(A, B, R, v, \sigma)$ value against $R, v$, and $\sigma$ :

$$
\mu(A, B):=\min _{R, v, \sigma} \operatorname{RMSD}(A, B, R, v, \sigma)
$$


Permutation $\sigma$ of degree $n$ is employed to consider all of the possible atom pairs to be compared between molecules $A$ and $B$.

If $R$ and $v$ are fixed, one finds a permutation $\sigma$ that minimizes RMSD efficiently by the Hungarian method, which was used in the context of measuring similarities of crystalline structures. ${ }^{39}$ Simultaneous optimization of $R, v$, and $\sigma$, however, seems to be much more difficult.

An efficient algorithm to find the permutation in $O\left(n^{4}\right)$ time was reported ${ }^{41}$ only in the case that molecules $A$ and $B$ coincide exactly when superimposed, which gives $\mu(A, B)=0$. However, to our best knowledge, an efficient algorithm to give the similarity indicator $\mu$ in the general case of $\mu(A, B) \neq 0$ has not been yet reported. The purpose of this study is to develop an efficient algorithm to calculate the similarity indicator $\mu$ of this generalized RMSD scheme. In other words, the purpose is to develop an efficient optimization algorithm to get an optimal superimposition for minimum RMSD without a unique atom mapping.

We now describe an algorithm for calculating the similarity indicator $\mu(A, B)$ in eq.(4). The translation vector $v$ can be easily determined as follows. Expanding $\operatorname{RMSD}(A, B, R, v, \sigma)$ in eq.(4), we have

$$
\begin{aligned}
\operatorname{RMSD}(A, B, R, v, \sigma)^{2} & \left.=\frac{1}{n} \sum_{i=1}^{n} \| a_{i}-R b_{\sigma(i)}-v\right) \|^{2} \\
& =\left\|v-\frac{1}{n} \sum_{i=1}^{n}\left(a_{i}-R b_{\sigma(i)}\right)\right\|^{2}+\Delta(A, B, R, \sigma),
\end{aligned}
$$

where the term $\Delta(A, B, R, \sigma)$ is independent of $v$. For any fixed $R$ and $\sigma$, RMSD is minimized at $v=v^{*}$ given by

$$
v^{*}:=\frac{1}{n} \sum_{i=1}^{n}\left(a_{i}-R b_{\sigma(i)}\right) .
$$

Therefore, to find an optimal choice of $(R, v, \sigma)$, one can first obtain $v=v^{*}$, and then search for the best pair of $R$ and $\sigma$ minimizing $\operatorname{RMSD}\left(A, B, R, v^{*}, \sigma\right)$.

Moving the origin of the coordinate axes to the centroids, one can represent $A$ and $B$ by $a_{i}^{\prime}=a_{i}-a_{g}(i=1,2, \ldots, n)$ and $b_{i}^{\prime}=b_{i}-b_{g}(i=1,2, \ldots, n)$, respectively, where $a_{g}=\frac{1}{n} \sum_{i=1}^{n} a_{i}$ and $b_{g}=\frac{1}{n} \sum_{i=1}^{n} b_{i}$. Then $v^{*}=\frac{1}{n} \sum_{i=1}^{n}\left(a_{i}^{\prime}-R b_{\sigma(i)}^{\prime}\right)=\mathbf{0}$ holds (Figure 1a). 
Therefore, the minimization problem reduces to computing

$$
\min _{R, \sigma}\left\{\sum_{i=1}^{n}\left\|a_{i}^{\prime}-R b_{\sigma(i)}^{\prime}\right\|^{2} \mid R: \text { orthogonal matrix, } \sigma: \text { permutation }\right\} \text {. }
$$

We henceforth discuss how to solve this minimization problem, assuming that the centroids of molecules $A$ and $B$ are located at the origin. The objective function in eq.(8) can be transformed as

$$
\begin{aligned}
\sum_{i=1}^{n}\left\|a_{i}-R b_{\sigma(i)}\right\|^{2} & =\sum_{i=1}^{n}\left(a_{i}^{\top} a_{i}+b_{\sigma(i)}^{\top} b_{\sigma(i)}-a_{i}^{\top} R b_{\sigma(i)}-b_{\sigma(i)}^{\top} R^{\top} a_{i}\right) \\
& =\sum_{i=1}^{n}\left(a_{i}^{\top} a_{i}+b_{\sigma(i)}^{\top} b_{\sigma(i)}\right)-2 \operatorname{trace}\left(R \sum_{i=1}^{n} b_{\sigma(i)} a_{i}^{\top}\right) \\
& =\sum_{i=1}^{n}\left(a_{i}^{\top} a_{i}+b_{i}^{\top} b_{i}\right)-2 \operatorname{trace}\left(R B P A^{\top}\right),
\end{aligned}
$$

where $P$ is a permutation matrix corresponding to $\sigma$, i.e., $P_{j i}=1$ if $j=\sigma(i)$ and $P_{j i}=0$ otherwise (Figure 1b). Eq.(10) comes from the commutativity of trace, i.e., trace $\left(R b a^{\top}\right)=$ $\operatorname{trace}\left(a^{\top} R b\right)=\operatorname{trace}\left(b^{\top} R^{\top} a\right)$. Since the first term of eq.(11) is a constant independent of $R$ and $P$, the minimization problem is tantamount to the maximization of trace $\left(R B P A^{\top}\right)$, i.e.,

$$
\max _{R, P}\left\{\operatorname{trace}\left(R B P A^{\top}\right) \mid R \text { : orthogonal matrix, } P \text { : permutation matrix }\right\} .
$$

Thus, the core part of this study is to develop an efficient algorithm to solve this maximization problem eq.(12).

We have implemented two heuristic algorithms to solve this problem, which are called Alternate Optimization (AO) and Tangent Space Relaxation (TSR) Methods. Details of these algorithms are described in the following sections.

\subsubsection{Alternate Optimization (AO) Method}

The optimization problem eq.(12) contains two variables, the orthogonal matrix (rotation) $R$ and the permutation matrix $P$. We consider optimizing this objective function associated with one of these variables while fixing the other one. This approach can reduce computational cost compared to optimizing for both $R$ and $P$ at the same time. 


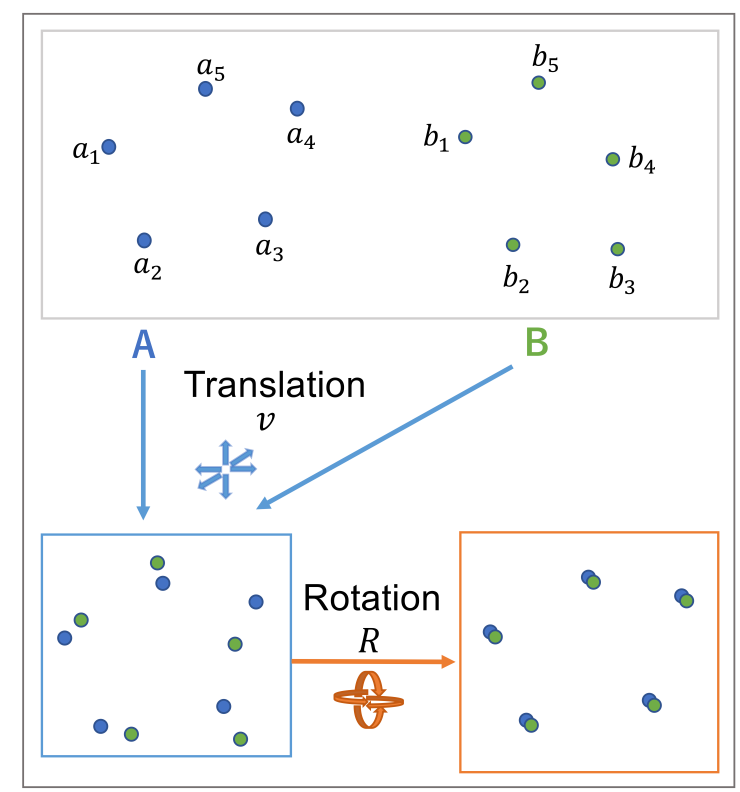

(a)

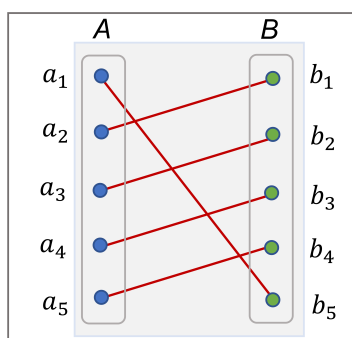

Atom mapping

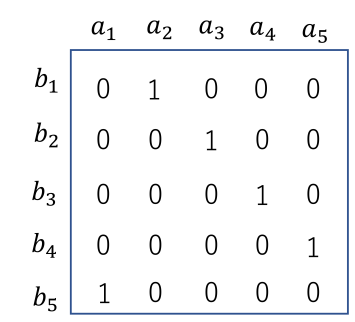

Permutation matrix $P$

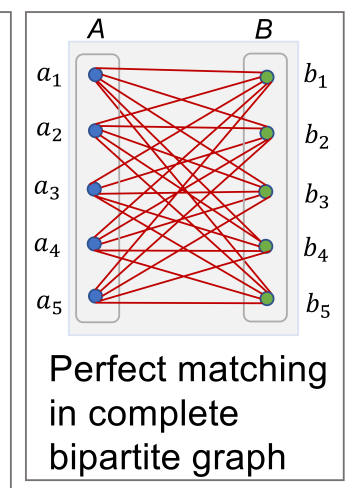

(c)

Figure 1: Graphical image of operations and matrix of G-RMSD algorithm. (a) Molecules $\mathrm{A}$ and $\mathrm{B}$ are translated with matrix $v$ and rotated with matrix $R$. Only atoms of $\mathrm{A}$ and $\mathrm{B}$ are shown. (b) Permutation matrix $P$ corresponding to a unique atom mapping between molecules A and B. (c) Perfect matching in complete bipartite graph. 
When the permutation matrix $P$ is fixed, meaning a fixed atom mapping, an optimal orthogonal matrix $R$ can be obtained efficiently by using the least-squares fitting method ${ }^{40}$ : If the singular valued decomposition of $B P A^{\top}$ is obtained as $U \Sigma V^{\top}$, the optimal solution is $R=V U^{\top}$.

The remaining question is how to find an optimal permutation matrix $P$ while the orthogonal matrix $R$ is fixed. The objective function trace $\left(R B P A^{\top}\right)$ is equal to trace $\left(P A^{\top} R B\right)$ because of the commutativity of trace. A permutation matrix $P$ corresponds to a perfect matching in the complete bipartite graph with vertex sets $A$ and $B$ (Figure 1c). Each edge between $a_{i} \in A$ and $b_{j} \in B$ is associated with a weight $w_{i j}:=a_{i}^{\top} R b_{j}$, which equals the $(i, j)$-component of $A^{\top} R B$. Then trace $\left(P A^{\top} R B\right)$ coincides with the sum of the edge weights in the perfect matching corresponding to $P$. Thus, maximizing the objective function reduces to an assignment problem, i.e., finding a perfect matching of maximum weight in the complete bipartite graph. The assignment problem can be solved efficiently by a combinatorial algorithm called the Hungarian method.

The AO method conducts the optimization of the objective function for $R$ and $P$ one after the other. A formal description of this method is given as follows.

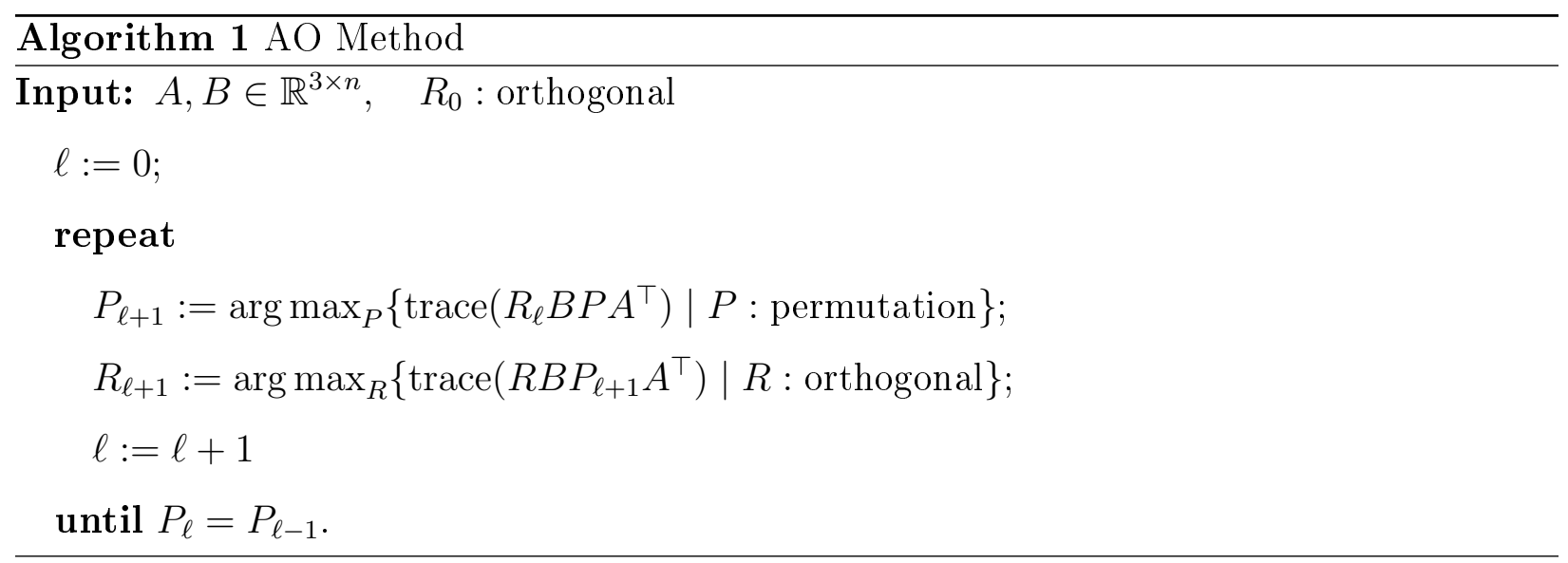

The bottleneck in each iteration is the Hungarian method, which takes $O\left(n^{3}\right)$ time. The number of iterations, however, is not bounded theoretically. It should also be noted that the algorithm may terminate with a local optimal solution that is not globally optimal. 


\subsubsection{Tangent Space Relaxation (TSR) method}

One possible way to avoid being trapped at a local optimal solution is to relax the condition for $P$ to be a permutation matrix. For instance, one can think of admitting $P$ to be an orthogonal matrix. Then the optimization of $P$ for a fixed $R$ can be done via the singular value decomposition instead of the Hungarian method. An apparent drawback of this approach is that it requires more computational cost. In addition, it is not clear how to get a permutation from the orthogonal matrix $P$ at termination. We then design an alternative approach called the TSR method.

The TSR method introduces a perturbation of the permutation matrix $P$ before conducting the optimization for $R$. The method fixes the orthogonal matrix $R$ and finds a permutation matrix $P$ that gives the optimal objective function. It then moves the $P$ matrix with the step length $\alpha>0$ in the tangent space of the manifold of all the orthogonal matrices at $P$ to the direction increasing the objective function. The perturbed permutation matrix obtained after this step is $\tilde{P}=P+\alpha S P$.

The problem of finding an optimal perturbation to eq.(12) is formulated as

$$
\max _{S}\left\{\operatorname{trace}\left(R B(P+\alpha S P) A^{\top}\right) \mid S: \text { skew-symmetric, }\|S\|_{\mathrm{F}}=1\right\} .
$$

Due to the linearity and commutativity of trace, this is tantamount to

$$
\max _{S}\left\{\operatorname{trace}\left(S P A^{\top} R B\right) \mid S: \text { skew-symmetric, }\|S\|_{\mathrm{F}}=1\right\} .
$$

The objective function is a linear combination of the upper triangular entries $s_{i j}(i<j)$ of the skew-symmetric matrix $S$. Let $c_{i j}$ denote the coefficient associated with $s_{i j}$. The constraint $\|S\|_{\mathrm{F}}=1$ can be reformulated as $\sum s_{i j}^{2}=\frac{1}{2}$. It then follows from the Cauchy-Schwarz inequality that

$$
\operatorname{trace}\left(S P A^{\top} R B\right)=\sum_{i, j} c_{i j} s_{i j} \leq \sqrt{\left(\sum_{i, j} c_{i j}^{2}\right)\left(\sum_{i, j} s_{i j}^{2}\right)}=\sqrt{\frac{1}{2}\left(\sum_{i, j} c_{i j}^{2}\right)},
$$

where the inequality is tight if and only if $s_{i j}$ is in proportion to $c_{i j}$. This enables us to compute an optimal skew-symmetric matrix $S$. In fact, it suffices to compute all $c_{i j}$ and to determine $s_{i j}$ in proportion to $c_{i j}$. Based on the skew-symmetric matrix $S$ thus determined, we update the orthogonal matrix $R$ so that $\operatorname{trace}\left(R B(P+\alpha S P) A^{\top}\right)$ is maximized. 
The resulting TSR algorithm is now described as follows. The computational cost for each iteration is again $O\left(n^{3}\right)$.

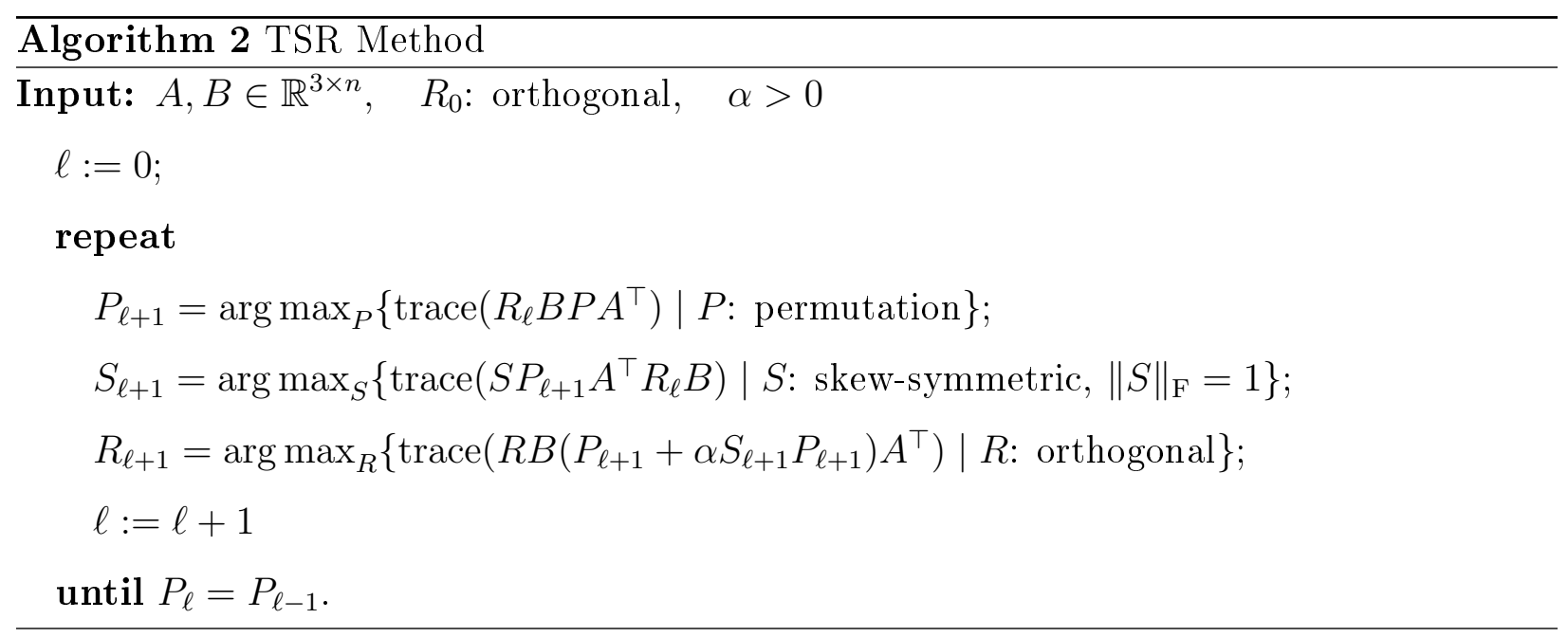

\subsection{Extension for partial structural comparison}

The AO method can be easily extended to compare molecules of different size.

We now consider a comparison between $A=\left\{a_{1}, \ldots, a_{m}\right\}$ and $B=\left\{b_{1}, \ldots, b_{n}\right\}$. Given an integer $k \leq m, n$, we define the silimarity indicator $\mu(A, B, k)$ as what follows. For subsets $I \subseteq\{1, \ldots, m\}$ and $J \subseteq\{1, \ldots, n\}$ of cardinality $k$, we denote $A_{I}:=\left\{a_{i} \mid i \in I\right\}$ and $B_{J}:=\left\{b_{j} \mid j \in J\right\}$. Let $\sigma$ be a bijection from $I$ to $J$, and put

$$
\operatorname{RMSD}(A, B, R, v, I, J, \sigma):=\sqrt{\frac{1}{k} \sum_{i \in I}\left\|a_{i}-R b_{\sigma(i)}-v\right\|^{2}} .
$$

The similarity indicator $\mu(A, B, k)$ is the minimum value of $\operatorname{RMSD}(A, B, R, v, I, J, \sigma)$ among all possible choices of $R, v, I, J$, and $\sigma$.

We now describe an extension of the $\mathrm{AO}$ method to compute $\mu(A, B, k)$. Given an orthogonal matrix $R$ and a transition vector $v$, one can find a triple $(I, J, \sigma)$ minimizing $\operatorname{RMSD}(A, B, R, v, I, J, \sigma)$ by solving a weighted bipartite matching problem. More specifically, consider a complete bipartite graph whose vertex sets are $A$ and $B$. Each edge between $a_{i}$ and $b_{j}$ has a weight $w_{i j}:=a_{i}^{\top} R b_{j}$. Find a maximum weight matching $M$ of cardinality $k$. This can be done in $O(k m n)$ time by a variant of the Hungarian method. Then the algorithm adopts the set of vertices in $A$ and $B$ incident to $M$ as $I$ and $J$, respectively. The matching 
$M$ naturally gives a bijection $\sigma$ between $I$ and $J$. The triple $(I, J, \sigma)$ is represented by a subpermutation matrix $P$ such that $P_{j i}=1$ if $j=\sigma(i)$ and $P_{j i}=0$ otherwise. With a fixed triple $(I, J, \sigma)$, one can obtain $R$ and $v$ minimizing $\operatorname{RMSD}(A, B, R, v, I, J, \sigma)$ via the singular value decomposition of $B P A^{\top}$. The extended AO method repeats this process.

A formal description of the extended AO method is as what follows, where $\mathbf{1}$ denotes the vector whose components are all one. The time complexity of each iteration is $O(\mathrm{kmn})$.

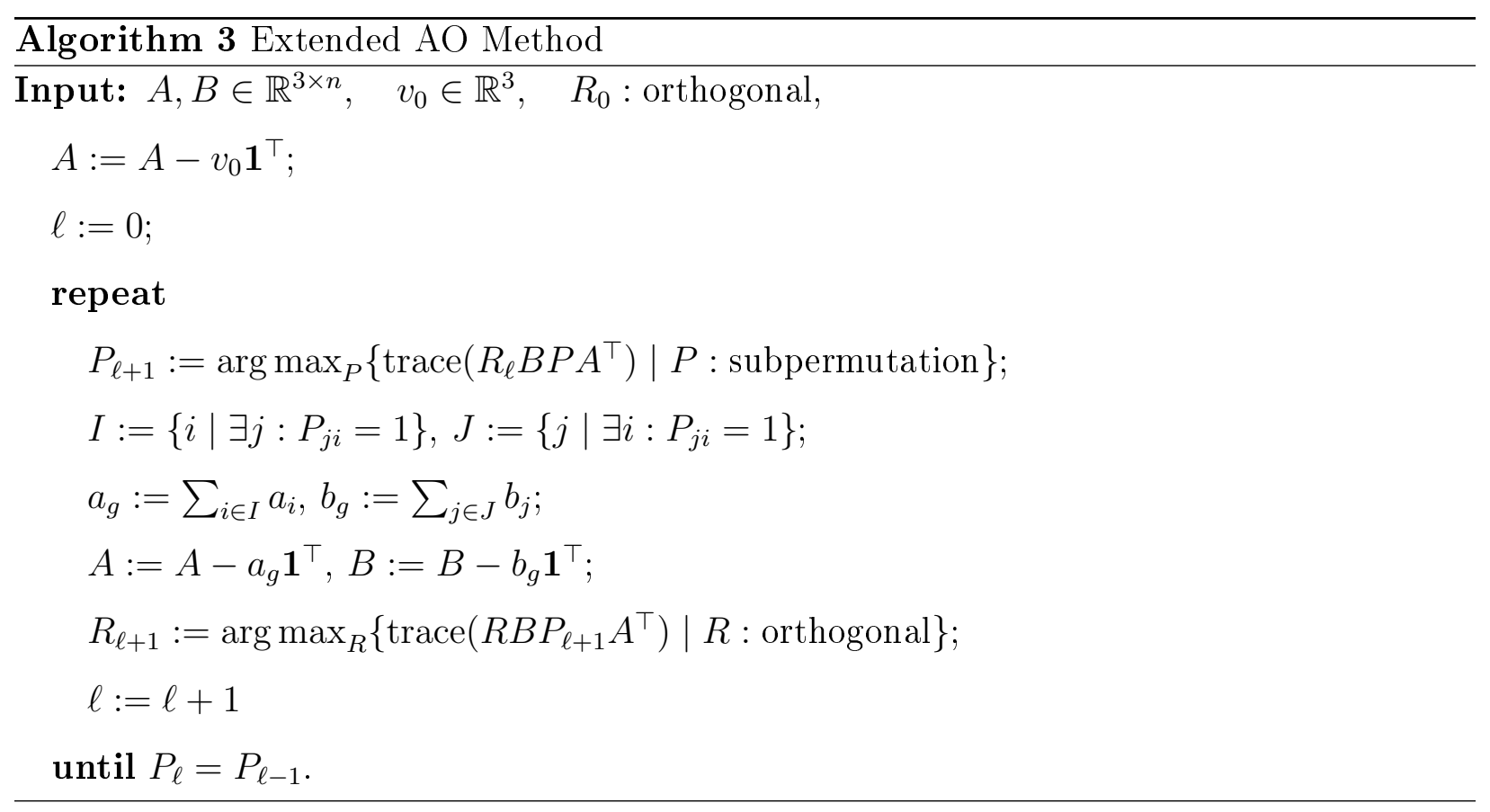

Similarly, one can extend the TSR method to this setting. Instead of the update of $R$ in Algorithm 3, the extended TSR method adopts the updates of $S$ and $R$ in Algorithm 2.

The comparison between the standard RMSD and G-RMSD is described in S1.1. of Supporting Information. The RMSD calculated by eq.(1) is called standard RMSD-1. The RMSD calculated by eq.(3) with fixed $R$ and $v$ is called standard RMSD-2. The difference is summarized in Table S1-1. 


\begin{tabular}{c|cccccccccc} 
Number of atoms & 10 & 20 & 30 & 40 & 50 & 60 & 70 & 80 & 90 & 100 \\
\hline The AO method & 3 & 6 & 5 & 8 & 4 & 4 & 4 & 7 & 4 & 7 \\
The TSR method & 8 & 8 & 7 & 11 & 4 & 5 & 5 & 7 & 6 & 7
\end{tabular}

Table 1: Number of correct answers (Initial $R_{0}$ : identity matrix)

\begin{tabular}{c|cccccccccc} 
Number of atoms & 10 & 20 & 30 & 40 & 50 & 60 & 70 & 80 & 90 & 100 \\
\hline The AO method & 94 & 85 & 93 & 92 & 84 & 91 & 88 & 86 & 89 & 91 \\
The TSR method & 98 & 94 & 96 & 94 & 92 & 97 & 95 & 93 & 95 & 94
\end{tabular}

Table 2: Number of correct answers (Initial orthogonal matrices: octahedral)

\section{RESULTS and DISCUSSION}

\subsection{Numerical experiment}

To evaluate the performance of the AO and TSR methods, we have conducted numerical experiments with artificial data, which are given by a random matrix $A$, a random orthogonal matrix $R^{*}$, and a random permutation matrix $P^{*}$. The matrix $B$ is determined by $B=$ $R^{*} A P^{*}$, which means $\mu(A, B)=0$. The parameter $\alpha$ in TSR method is set as $\alpha=0.3$.

Table 1 shows the number of success instances among randomly generated 100 test data sets when the initial orthogonal matrix $R_{0}$ is fixed to be the identity matrix.

Tables 2 and 3 exhibit the same kind of results when we adopt the best solution among different trials with systematically constructed initial orthogonal matrices. Table 2 adopts the 48 orthogonal matrices that keep the regular octahedron invariant, whereas Table 3 adopt the 120 orthogonal matrices that keep the regular icosahedron invariant. These initial matrices are chosen so that one can start with an unbiased set of rotations.

Experimental results in Tables 1-3 show that the icosahedral setting of initial orthogonal matrices leads to accurate computation of $\mu(A, B)$ both in the AO and TSR methods. There are some instances for which TSR works while AO fails. However, the running time of AO is shorter than that of TSR.

The dataset used for the numerical experiment is available at the project home page at https://github.com/striwata/GRMSD. 


\begin{tabular}{c|cccccccccc} 
Number of atoms & 10 & 20 & 30 & 40 & 50 & 60 & 70 & 80 & 90 & 100 \\
\hline The AO method & 100 & 100 & 100 & 100 & 100 & 100 & 100 & 100 & 100 & 100 \\
The TSR method & 100 & 100 & 100 & 100 & 100 & 100 & 100 & 100 & 100 & 100
\end{tabular}

Table 3: Number of correct answers (Initial $R_{0}$ : icosahedral)

\subsection{Applications to molecular data}

The performance tests with artificial data in the previous section showed that both AO and TSR robustly give accurate results, although TSR sometimes gives slightly better results. Calculation cost of AO is less than that of TSR. Therefore, we have used the AO method for further applications to molecular data.

We chose two types of dataset obtained by QM-calculations to demonstrate the performance of G-RMSD. The first application is to show similarity determination between 3D-valence structures with the same number of atoms without any specifications other than the Cartesian coordinates of atoms. We did calculations for a dataset of 653 conformers of $\alpha$-D-glucose. Similarity of conformation of pyranose rings was measured for all the combinations of glucose structures. We have analyzed the correlations between the G-RMSD values $\mu$ and sugar conformer types. A clustering of glucose structures using the G-RMSD similarity will be shown.

The second application is to 3D-database search, which cannot be done by standard RMSD due to the restriction of unique atom mapping or superimposition (Table S1-1). This application demonstrates the similarity determination between structures with different number of atoms without any specifications other than the Cartesian coordinates of atoms. It is also to show performance of G-RMSD for non-valence and TS structures. The dataset contains 689 3D-isomers of $\mathrm{C}_{2} \mathrm{H}_{4} \mathrm{O}_{2}$. The dataset was searched with four types of 3D-query.

These two datasets were obtained by exploration of QM potential energy surface using the Scaled Hypersphere Search of the Anharmonic Downward Distortion Following (SHS-ADDF) method $^{42-45}$ within the GRRM14 software program package. ${ }^{46}$ The datasets used here are available as Supporting Information and also from the r-map database via RMapServer. ${ }^{29}$ 


\begin{tabular}{c|ccc} 
structure-pair groups & $\mathrm{a}$ & $\mathrm{b}$ & $\mathrm{c}$ \\
\hline sample numbers & 31506 & 43439 & 137933 \\
average & 0.0356 & 0.0656 & 0.2844 \\
variance & 0.0013 & 0.0031 & 0.012 \\
standard deviation & 0.0364 & 0.0559 & 0.1096 \\
median & 0.028 & 0.0624 & 0.3269 \\
the most frequent value & $0.0214 \pm 0.0011$ & $0.0747 \pm 0.0011$ & $0.3425 \pm 0.0011$ \\
maximum & 0.878 & 0.8886 & 0.9015 \\
minimum & 0.0006 & 0.0076 & 0.0089
\end{tabular}

Table 4: Statistics of $\mu$ Values for Structure-pair Groups of $\alpha$-D-Glucose (1). (a) Structurepairs adopting the same conformer type and superimposed with the same atom types, (b) Structure-pairs adopting the same conformer type and superimposed partly with different atom types (e.g., two pairs of carbon and oxygen atoms are in a superimposed structure), (c) Structure-pairs adopting different conformer types.

\subsubsection{Similarity of glucose conformers}

The relationship between the sugar ring conformation and the reactivity/selectivity of glycosylation reactions is one of the topics intensively studied in current glycochemistry. ${ }^{47-56}$ Theoretical and experimental efforts have been devoted to revealing the influence of change of sugar ring conformations during reactions. In these analyses, determining the similarity of sugar ring conformations is an essential step.

We will demonstrate classification of $\alpha$-D-glucose (1) conformers (Figure 2) on the basis of similarity determination by using G-RMSD. In this study, we simplified the question by focusing only on the 3D-similarity of the six-membered rings (pyranose rings) consisting of C-1, C-2, C-3, C-4, C-5, and O.

The nomenclature of the six-membered (pyranose) and furanose (five-membered) ring conformations is defined on the basis of the IUPAC rules for six- and five-membered rings ${ }^{57-59}$. For cyclohexane, a typical six-membered ring structure with six equivalent carbon atoms, five distinct conformers are defined: chair $(C)$, boat $(B)$, twist/skew-boat $(S)$, half-chair $(H)$, and envelope $(E)$ conformations (Figure 2a). These five distinct pyranose ring conformers 
<smiles>OCC1O[C@H](O)C(O)[C@H](O)[C@H]1O</smiles>

1

(a)

(b)

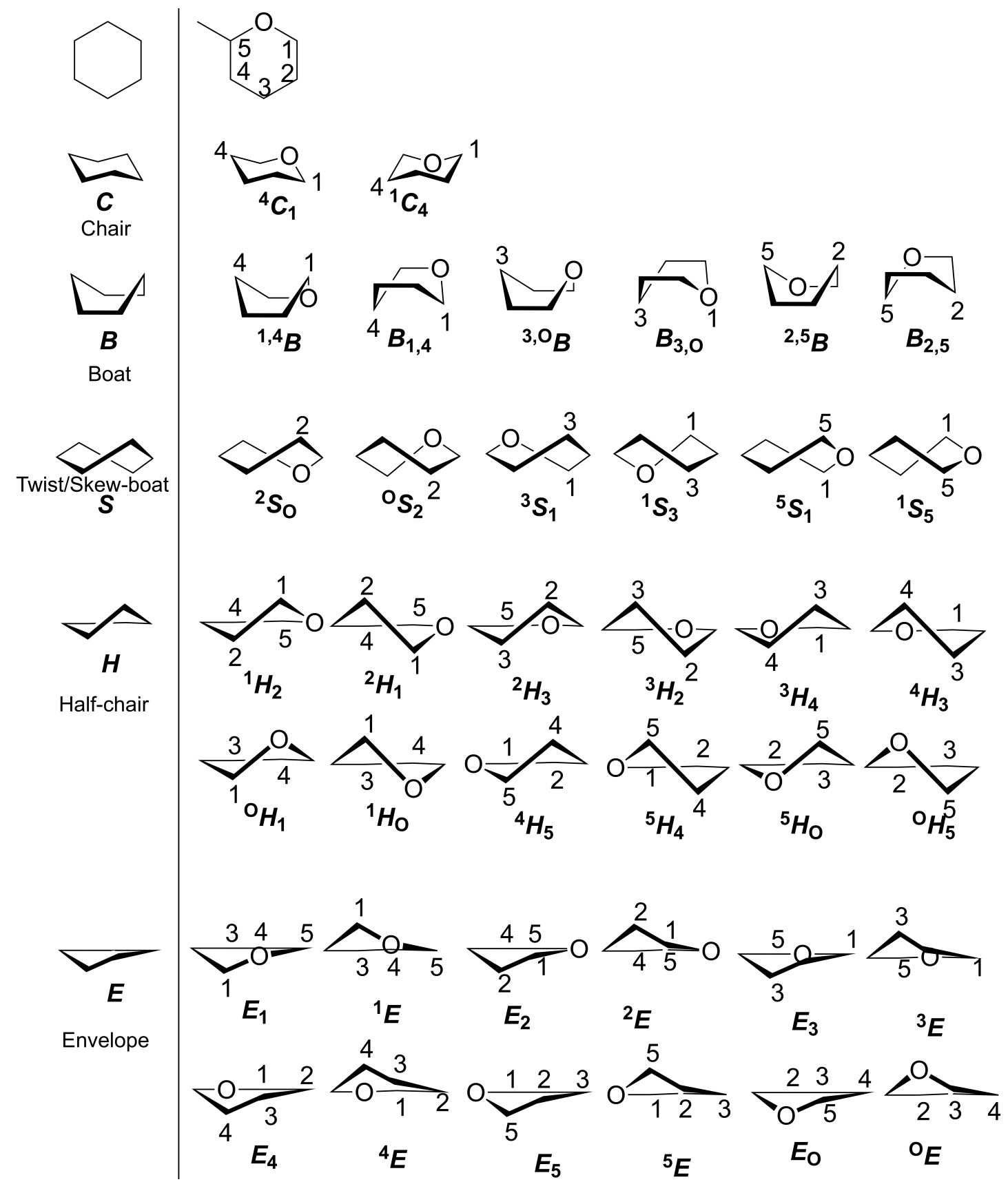

Figure 2: $\alpha$-D-glucose (1) and IUPAC nomenclature of six-membered ring conformers. 
are further categorized into 38 types in total by adding the serial numbers of carbon atoms to those conformer notations according to the relative positions compared to a reference plane (Figure 2b). The conformers are described numerically by using Cremer-Pople puckering coordinates (CP coordinates) ${ }^{60,61}$, which is a mathematical method to describe 3D-form of a hexagon and is commonly used for the notation of pyranose rings. The puckering coordinates $(\theta, \phi, r)$ can be calculated from Cartesian coordinates of the ring atoms.

Since we focused on the geometry of six-membered rings, some of the conformer types in Figure $2 \mathrm{~b}$ are categorized into the same group. For example, the ${ }^{4} C_{1}$ and ${ }^{1} C_{4}$ conformers are in principle the same 3D-geometry group (Figure S1-1), although bond lengths and angles of the ring can be slightly different because of the difference of orientation of substituents (e.g., axial or equatorial hydroxyl groups).

The dataset is composed of 653 (209 EQ, 443 TS, and 1 DC) structures, calculated by a conformational search using the SHS-ADDF method at the RHF/6-31G level previously reported by some of the authors. ${ }^{56}$ The conformational search was conducted by tracing only low TS barriers. Its output files, which we have used in this study, usually contain mostly conformers and sometimes include some bond-breaking channels. The numbers of bond-breaking channels in this case were 17 (8 EQ, 8 TS, and 1 DC). See ref. ${ }^{56}$ for more detailed information on the dataset.

We calculated 3D similarity indicator $\mu$ between all the pairs of the structures by focusing on six-membered rings. The similarity $\mu$ was calculated using the Cartesian coordinates of the six atoms without a unique atom mapping between the molecules. Information about atom and bond types was not used either. Note that smaller $\mu$ value means that the pair of the structures is more similar. We analyzed the results by annotating each conformer by using the $\mathrm{CP}$ coordinates. We calculated the $\mathrm{CP}$ coordinates using the program developed by Whitfield et al. ${ }^{62,63}$ and classified them into the five conformer types for the analysis.

Figure 3 shows the distribution of the calculated $\mu$ values for $\mathbf{1}$. The statistics analysis is shown in Table 4. The structure pairs are categorized into three groups and indicated in different colors: (a) those adopting the same conformer type and superimposed with the same atom types, colored blue; (b) those adopting the same conformer type and superimposed partly with different atom types (e.g., two pairs of carbon and oxygen atoms are in a 


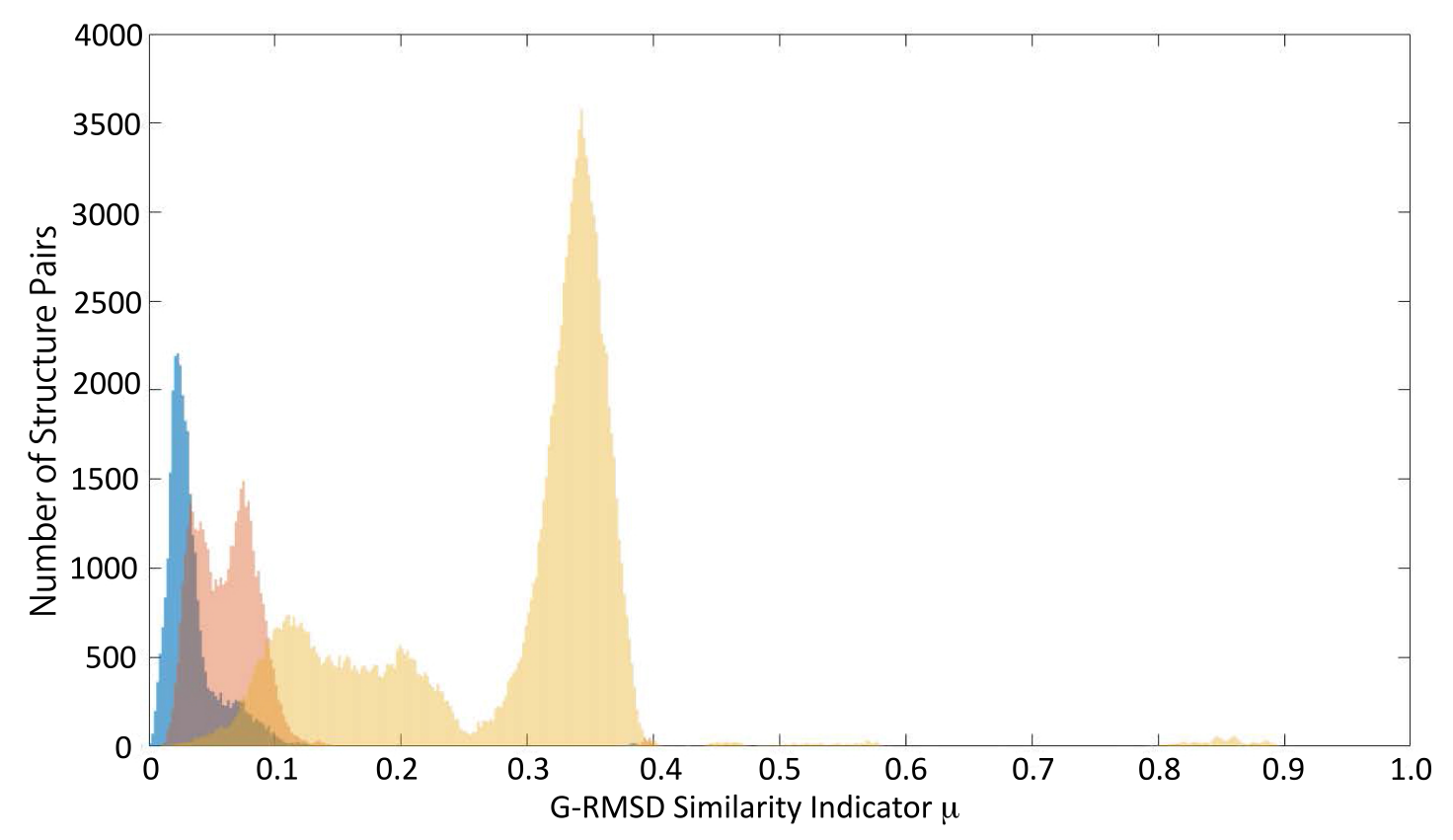

Figure 3: Distribution of G-RMSD similarity indicator $\mu$ for $\alpha$-D-glucose (1). Blue distribution shows structure pairs with same conformers and fully matched atom types. Red distribution shows structure pairs with same conformers and partly nonmatched atom types. Yellow distribution shows structure pairs with different conformer types.

superimposed structure), colored red; and (c) those adopting different conformer types, colored yellow. These three groups are well categorized in the histograms. A smaller $\mu$ value means that the pair has higher similarity. The ordering of the groups in the histogram is $\mathrm{a} \rightarrow \mathrm{b} \rightarrow \mathrm{c}$ from small $\mu$ values. These results demonstrate that the similarity of the conformations are well distinguished with the $\mu$ values.

Then, we have performed classification of the conformers for $\mathbf{1}$ based on the similarity of the pyranose ring conformations using the $\mu$ values. The classification has been done by using the EQ conformers. The distance between the structures was calculated based on an attractive force, which was defined to be in proportion to $\frac{1}{\mu}$. Therefore, a larger attractive force, which leads to a closer distance, was assigned between structures with higher similarity. We have used the Gephi software ${ }^{64}$ to visualize the results of the classification.

The results of the classification demonstrate that the $\mu$ values accurately detected the similarity of the pyranose ring conformations as shown in Figure 4: 

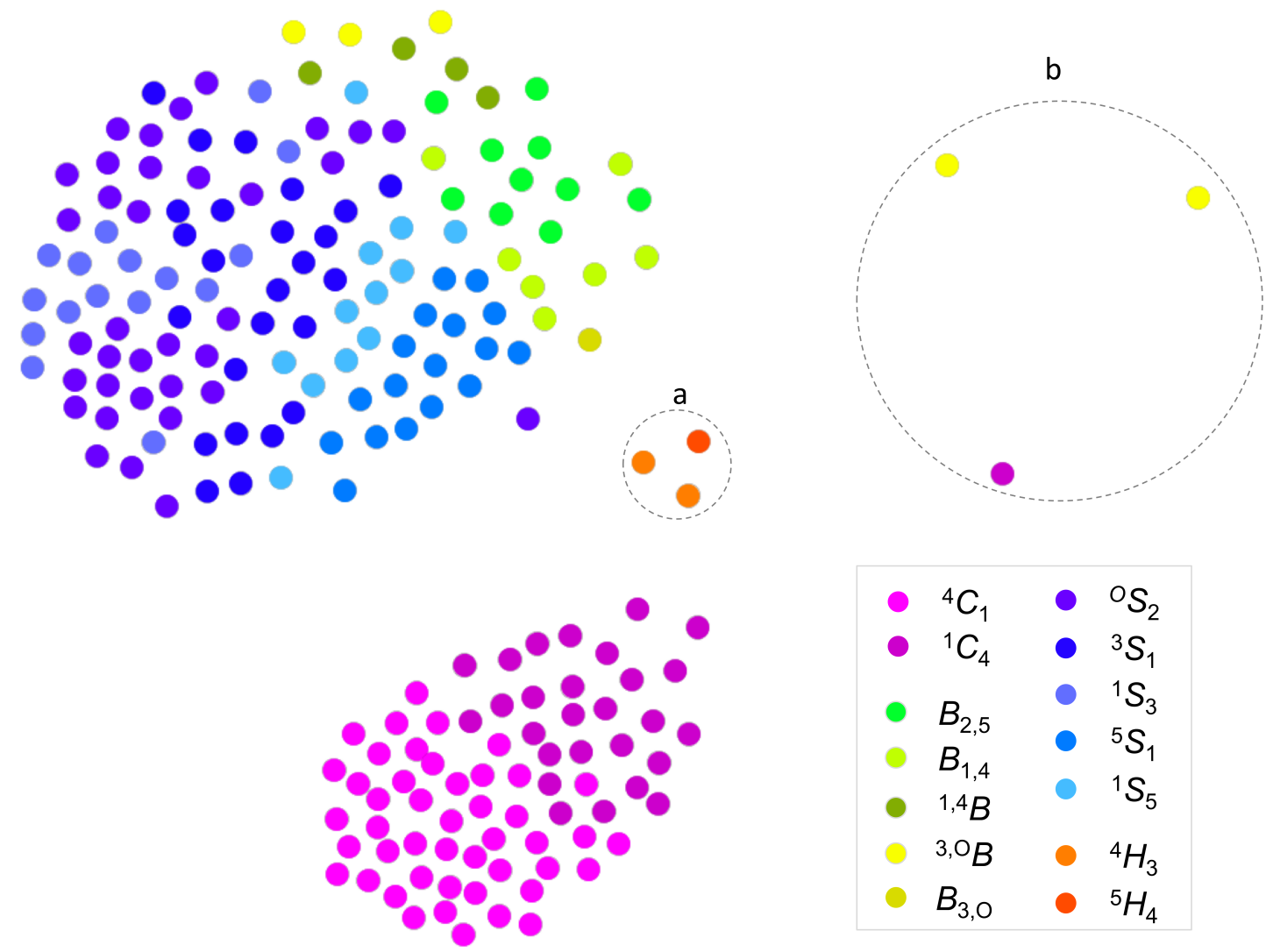

$\begin{array}{ccc}{ }^{4} C_{1} & \bullet & { }^{0} S_{2} \\ { }^{1} C_{4} & \bullet & { }^{3} S_{1} \\ B_{2,5} & \ddots & { }^{1} S_{3} \\ B_{1,4} & \ddots & { }^{5} S_{1} \\ { }^{1,4} B & \ddots & { }^{1} S_{5} \\ { }^{3,0} B & \ddots & { }^{4} H_{3} \\ B_{3,0} & \bullet & { }^{5} H_{4}\end{array}$

Figure 4: Classification of pyranose ring conformations of $\alpha$-D-glucose (1) using similarity indicator $\mu$. Each dot in the plot corresponds to a glucose conformer. The distance between the conformers was calculated based on an attractive force, which was defined to be in proportion to $\frac{1}{\mu}$. If $\mu=0$, the force is not calculated, and it is determined that they are the same. A larger attractive force leads to a closer distance between structures, meaning higher similarity. The similarity information is completely contained in the distances. The group (a) contains all the $H$ half-chair conformers. These conformers were structures obtained after water elimination from $\alpha$-D-glucose. The group (b) contains products from the endo-cyclic cleavage reaction. 
The chair $(C)$, boat $(B)$, twist/skew-boat $(S)$, and half-chair $(H)$ conformations are magenta-pinkish, green-yellowish, blueish, and orangish colored, respectively. All of the structures in the $H$ half-chair conformations, which are in circle a, were structures obtained after water elimination from $\alpha$-D-glucose. The structures produced via an endo-cyclic cleavage reaction, that means, one of the bonds in the pyranose ring was cleaved, are in circle $\mathrm{b}$. The other structures, where no bond broke, were grouped into two clusters. One of the clusters is composed of the $C$ chair conformations, and the other cluster is composed of the $B$ boat and $S$ twist/skew-boat conformations. This clustering is reasonable because the geometry of the two types of the $C$ chair conformation is the same and only differs by the relative position of the carbon and oxygen atoms. The geometry is different from those of $B$ boat and $S$ twist/skew-boat conformations as can be seen in Figure 2. The $B$ boat and $S$ twist/skewboat conformations form a cluster. This clustering result is in good agreement with the fact that the geometries of the $B$ boat and $S$ twist/skew-boat conformations are similar. For example, the twisted form of a ${ }^{1,4} \mathrm{~B}$ boat conformation against the $\mathrm{C} 2-\mathrm{C} 3$ and $\mathrm{C} 5$-O bonds is a ${ }^{2} S_{O}$ twist/skew-boat conformation. The ${ }^{4} C_{1}$ and ${ }^{1} C_{4}$ chair conformations form one group and are clearly distinguished. This means, G-RMSD recognized that the ${ }^{4} C_{1}$ and ${ }^{1} C_{4}$ conformers are very similar. The $B$ boat and $S$ twist/skew-boat conformations are also clearly grouped. The difference of the relative position of the atoms was not very clearly distinguished, e.g., ${ }^{O} S_{2}$ and ${ }^{1} S_{3}$, but these similar conformations are closely located.

Concerning the comparison with the standard RMSD, we illustrated in Figure S1-2 the difference between standard RMSD-1 and G-RMSD in 3D-similarity measuring ${ }^{4} C_{1}$ and ${ }^{1} C_{4}$ six-membered rings. As shown in Figure S1-2, standard RMSD-1 cannot achieve the correct superimposition due to the restriction of the unique atom mapping. G-RMSD can achieve the correct superimposition by rotating and translating the two ring position and by considering the permutation of atom-pair mapping. Standard RMSD-2 cannot be applied to this measurement because of the restriction of superimposition.

\subsubsection{Partial structure search of global reaction map of $\mathrm{C}_{2} \mathrm{H}_{4} \mathrm{O}_{2}$}

The dataset for the demonstration of 3D-database search was obtained by an exhaustive exploration of potential energy surface for $\mathrm{C}_{2} \mathrm{H}_{4} \mathrm{O}_{2}$ with SHS-ADDF at the B3LYP/6-31G(d) 


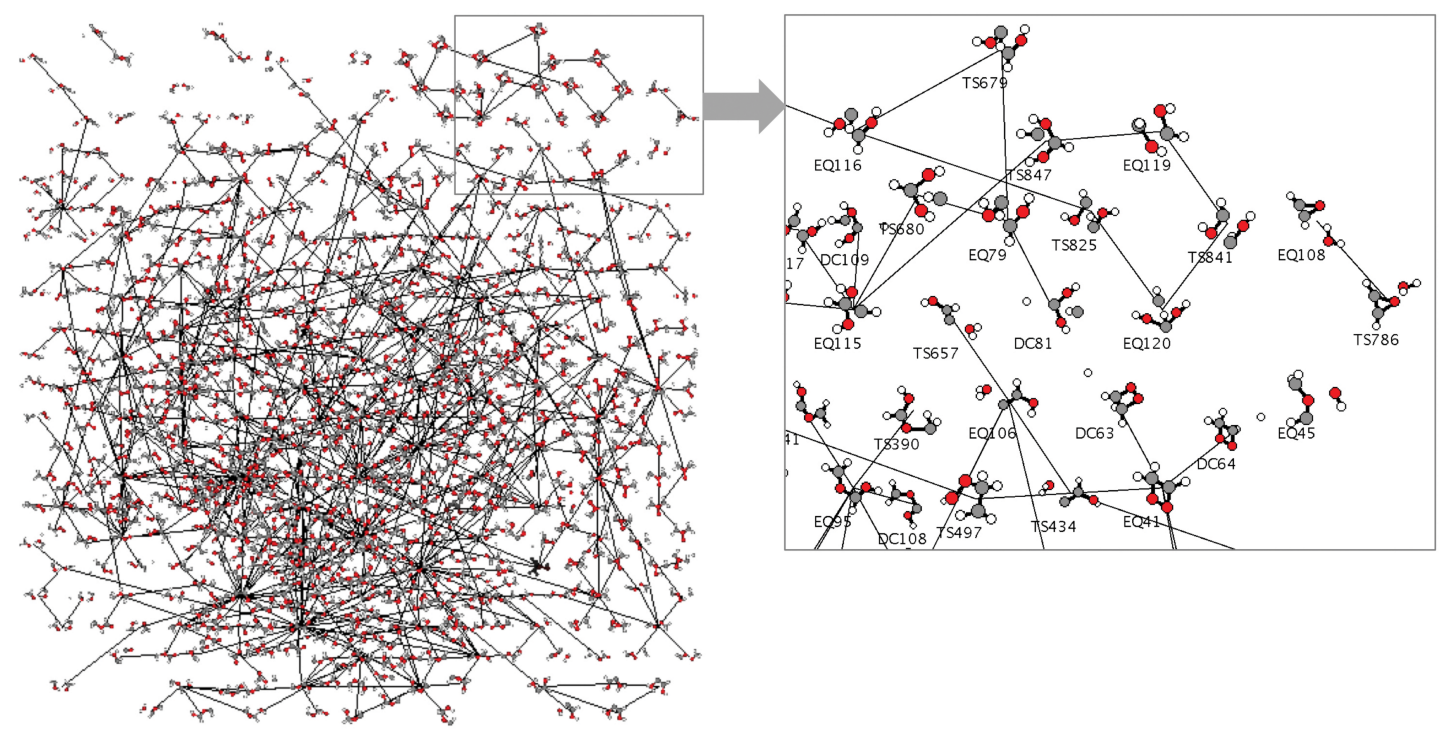

Figure 5: Global r-map of $\mathrm{C}_{2} \mathrm{H}_{4} \mathrm{O}_{2}$. Overview of the full map (left) and part of the map (right). The r-map is composed of $121 \mathrm{EQ}, 457 \mathrm{TS}$, and $111 \mathrm{DC}$ structures, which are connected with IRC.

level, which contains all possible reactions composed of its input compositional formula ${ }^{65-67}$. It is composed of 689 (121 EQ, $457 \mathrm{TS}$, and $111 \mathrm{DC}$ ) structures, which are connected via IRC. Figure 5 shows the global r-map visualized with RMapViewer. ${ }^{27,56,68}$ This r-map contains reactions from acetic acid $\left(\mathrm{CH}_{3} \mathrm{COOH}\right)$ to ethylenediol $\left(\mathrm{CH}_{2} \mathrm{C}(\mathrm{OH}) \mathrm{OH}\right)$ and from acetic acid to glycoaldehyde $\left(\mathrm{CH}_{2}(\mathrm{CHO}) \mathrm{OH}\right)$, for example.

We searched the r-map dataset with four types of 3D-query: $\mathrm{CH}_{3}$ anion (2), $\mathrm{CH}_{3}$ cation (3), $\mathrm{CH}_{2} \mathrm{OH}$ anion (4), and $\mathrm{CH}_{2} \mathrm{OH}$ cation (5) (Figure 6). The structures were optimized at the B3LYP/6-31G(d) level by using the Gaussian 16 program package. ${ }^{69}$ The geometry of the queries are shown in Figure 4. The two types of $\mathrm{CH}_{3}$ structures $\mathbf{2}$ and $\mathbf{3}$ were optimized to different geometry: $\mathbf{2}$ adopts a trigonal pyramid structure, whereas $\mathbf{3}$ adopts a planer structure. The optimized structures of the two types of $\mathrm{CH}_{2} \mathrm{OH} \mathbf{4}$ and $\mathbf{5}$ were non-planer and planer structures, respectively. Rather different C-O bond lengths were found for $\mathbf{4}$ and 5, namely, 1.51 and $1.25 \AA$, respectively.

Figure 7 shows the distribution of the G-RMSD similarity indicator $\mu$ values between $\mathrm{C}_{2} \mathrm{H}_{4} \mathrm{O}_{2}$ (689 structures) and $\mathrm{CH}_{3}$ anion (2), whose geometry is similar to that of a usual neutral methyl group. Small $\mu$ values were obtained for the $\mathrm{C}_{2} \mathrm{H}_{4} \mathrm{O}_{2}$ structures bearing 
<smiles>CC=[V]C</smiles>

\begin{tabular}{cccc} 
Structure no. & $\begin{array}{c}\gamma 1 \\
{[\mathrm{deg}]}\end{array}$ & $\begin{array}{c}\mathrm{C}-\mathrm{H} \\
{[\AA]}\end{array}$ & $\begin{array}{c}\theta 1 \\
{[\mathrm{deg}]}\end{array}$ \\
\hline $\mathbf{2}$ & 46.6 & 1.13 & 100.0 \\
$\mathbf{3}$ & 0.0 & 1.09 & 120.0
\end{tabular}

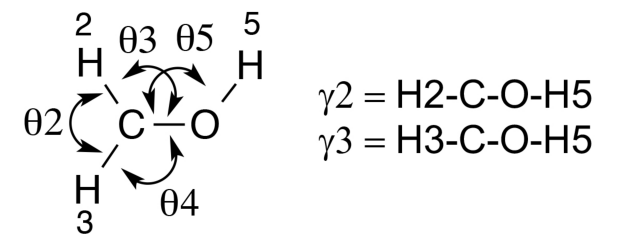
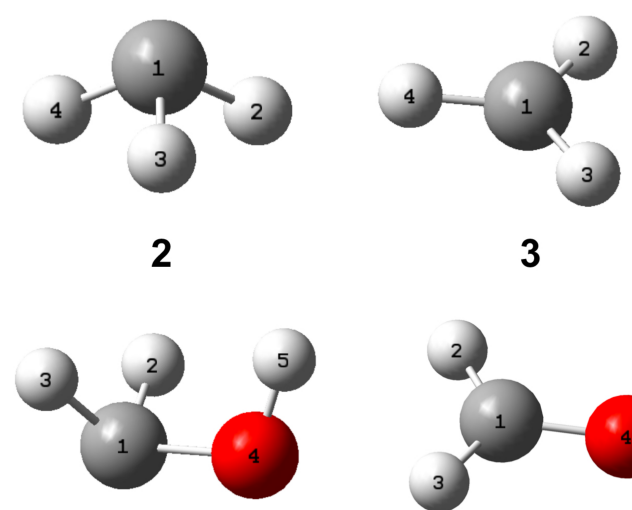

4

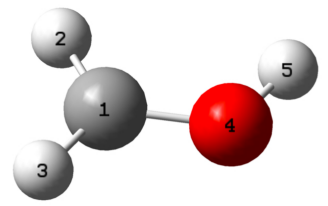

5

\begin{tabular}{cccccccccc} 
Structure no. & $\begin{array}{c}\gamma 2 \\
{[\mathrm{deg}]}\end{array}$ & $\begin{array}{c}\gamma 3 \\
{[\mathrm{deg}]}\end{array}$ & $\begin{array}{c}\mathrm{C}-\mathrm{H} \\
{[\AA]}\end{array}$ & $\begin{array}{c}\mathrm{C}-\mathrm{O} \\
{[\AA \AA}\end{array}$ & $\begin{array}{c}\mathrm{O}-\mathrm{H} \\
{[\AA \AA}\end{array}$ & $\begin{array}{c}\theta 2 \\
{[\mathrm{deg}]}\end{array}$ & $\begin{array}{c}\theta 3 \\
{[\mathrm{deg}]}\end{array}$ & $\begin{array}{c}\theta 4 \\
{[\mathrm{deg}]}\end{array}$ & $\begin{array}{c}\theta 5 \\
{[\mathrm{deg}]}\end{array}$ \\
\hline $\mathbf{4}$ & 52.0 & 52.0 & 1.14 & 1.51 & 0.98 & 99.7 & 104.3 & 104.3 & 109.8 \\
$\mathbf{5}$ & 0.0 & 0.0 & 1.09 & 1.25 & 0.99 & 122.4 & 122.0 & 115.6 & 116.1
\end{tabular}

Figure 6: Structures and geometry of 3D-queries to search r-map dataset: $\mathrm{CH}_{3}$ anion (2), $\mathrm{CH}_{3}$ cation (3), $\mathrm{CH}_{2} \mathrm{OH}$ anion (4), and $\mathrm{CH}_{2} \mathrm{OH}$ cation (5). Both of the cations 3 and 5 adopt planer structures. 


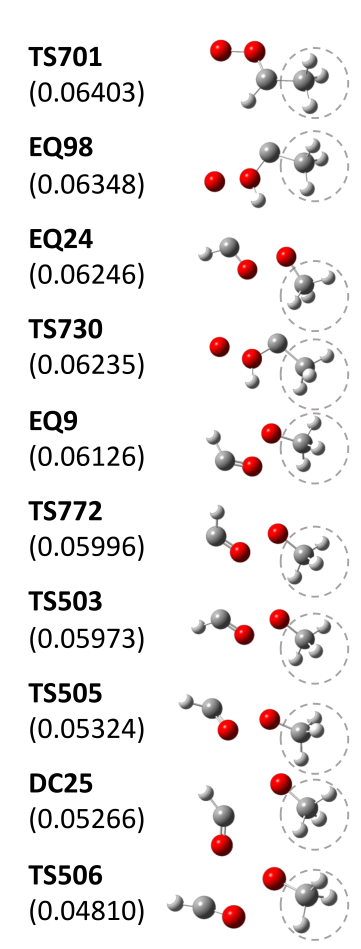

(a)

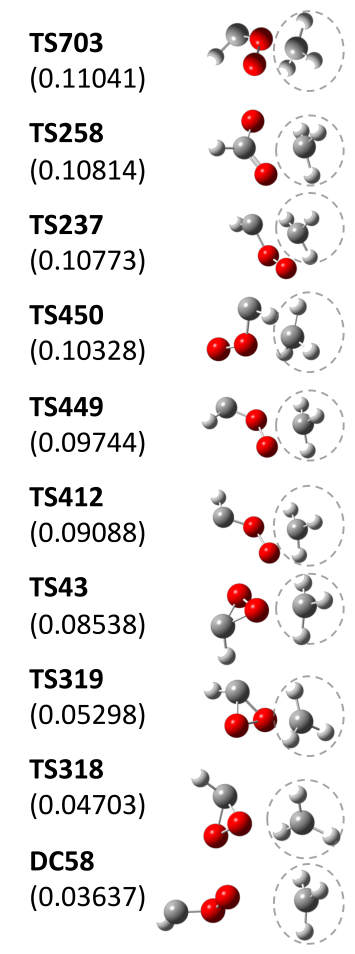

(b)

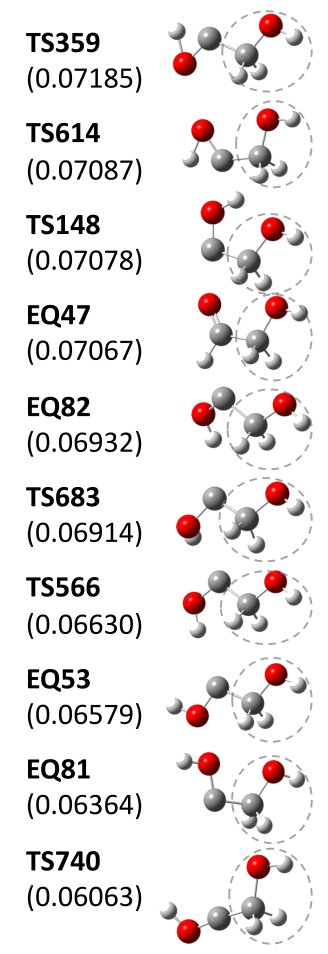

(c)

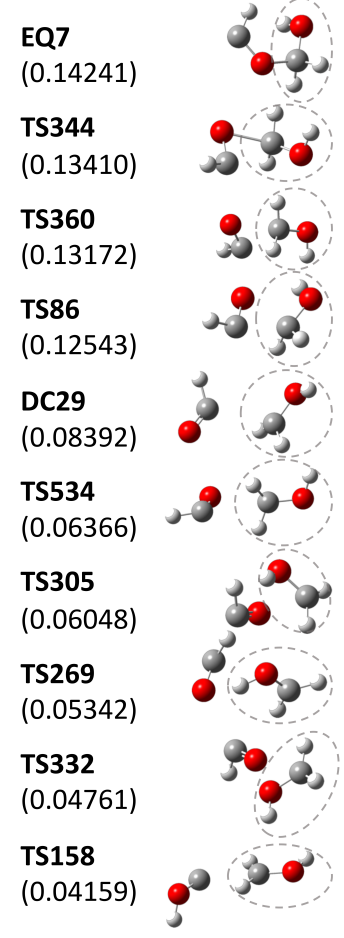

(d)

Figure 7: Top ten structures with high similarity to query structures (a) 2, (b) 3, (c) 4, and (d) 5 .

methyl group(s) (the histogram in blue), whereas larger $\mu$ values were obtained for those without methyl group (the histogram in red). The blue and red histograms are clearly separated. This result shows that the similarity of partial structures can be correctly recognized with the $\mu$ values.

The top ten structures that are similar to each of the 3D-queries $\mathbf{2 - 5}$ are shown at the bottom in Figure 8. The structure ID denotes the type of structure (EQ, TS, or DC) and its serial no., e.g., TS701 means that it is a TS structure with the serial no. 701. The number in parenthesis is the $\mu$ value. Partial structures matched with the query were shown in the dotted line. The differences from the query in geometry for those structures as well as the $\mu$ values are shown in Figures 9-12.

Only rather small differences were found in the geometry between the matched methyl group of all the top ten structures and query $\mathbf{2}$, and there is a good correlation between the differences and the $\mu$ values (Figures $8 \mathrm{a}$ and 9 ). The first five structures with $\mu$ values less 


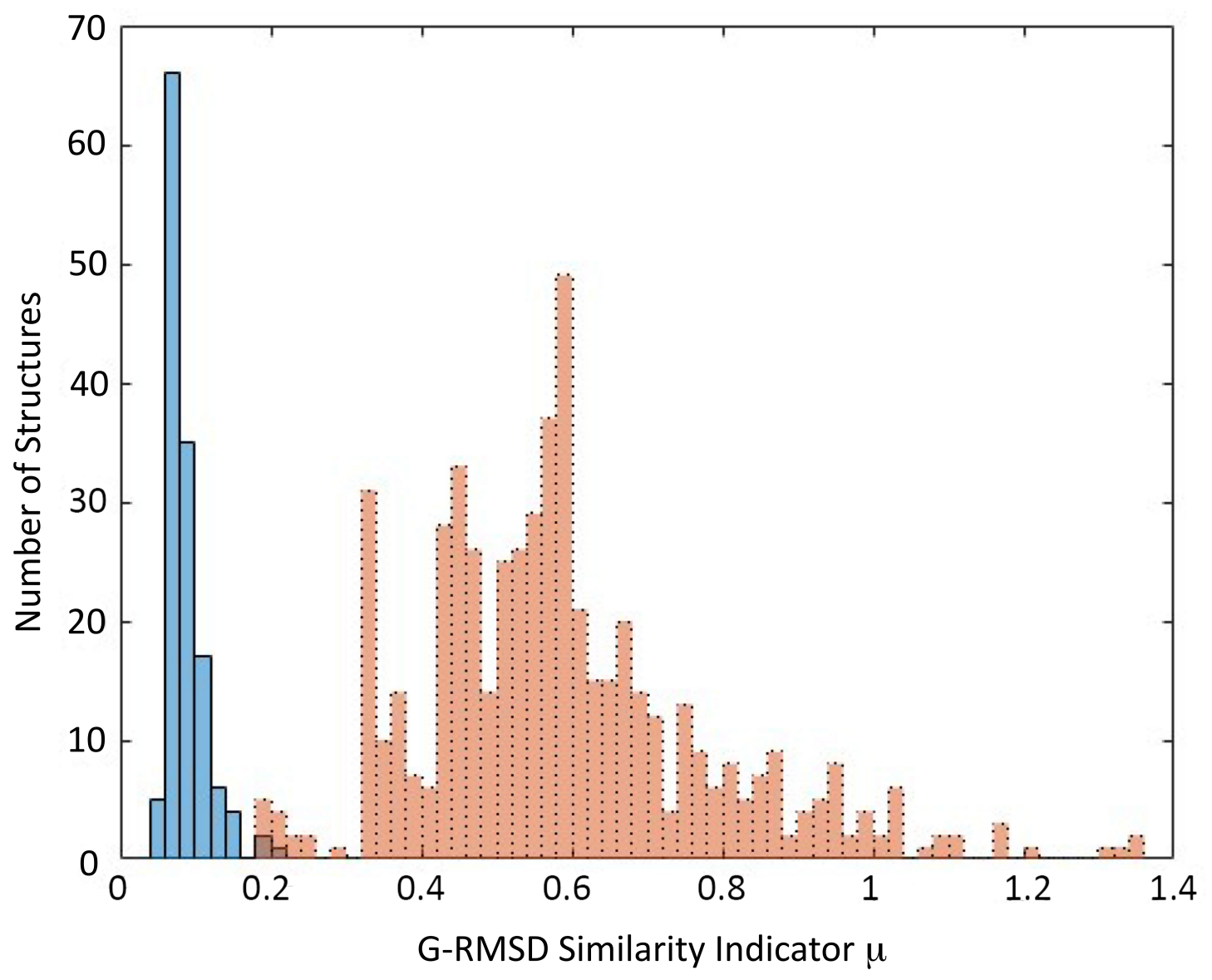

Figure 8: Distribution of G-RMSD similarity indicator $\mu$ between $\mathrm{CH}_{3}$ anion (2) and $\mathrm{C}_{2} \mathrm{H}_{4} \mathrm{O}_{2}$ isomers (689 structures). Blue: $\mathrm{C}_{2} \mathrm{H}_{4} \mathrm{O}_{2}$ structures with methyl group(s). Red: $\mathrm{C}_{2} \mathrm{H}_{4} \mathrm{O}_{2}$ structures without methyl group. 

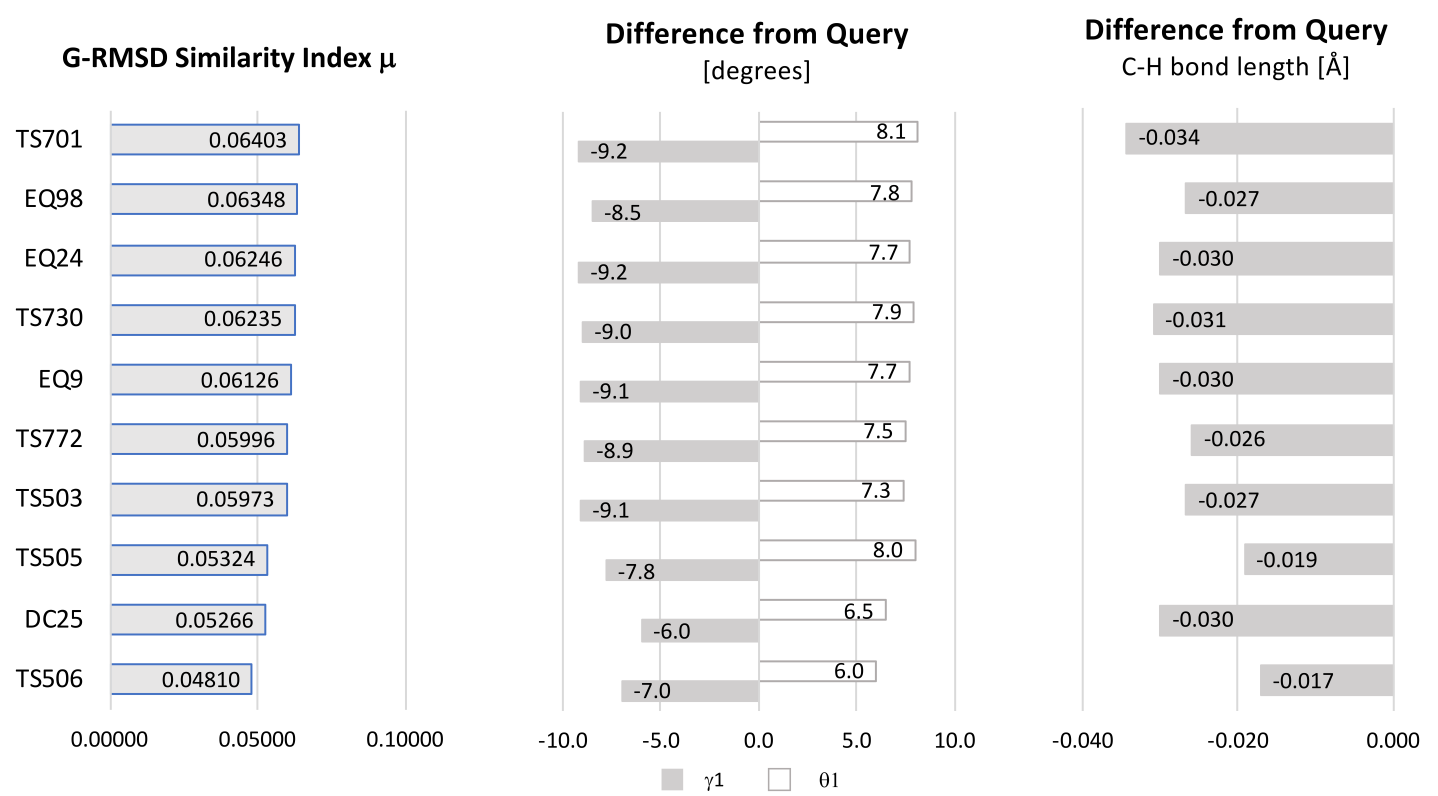

Figure 9: G-RMSD similarity indicator $\mu$ of structures with high similarity to query structure 2 and geometry differences.
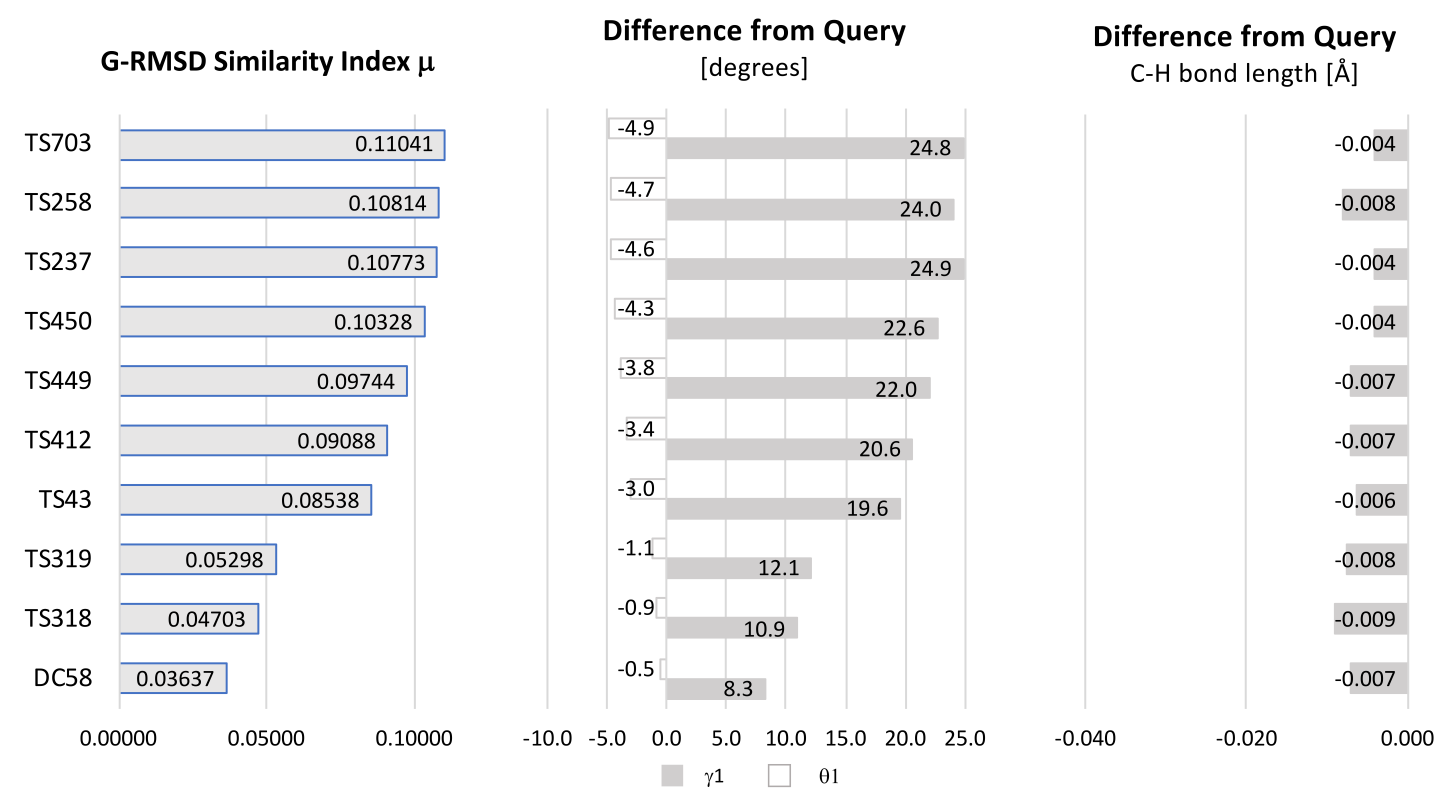

Figure 10: G-RMSD similarity indicator $\mu$ of structures with high similarity to query structure $\mathbf{3}$ and geometry differences. 


\section{G-RMSD Similarity Index $\mu$}
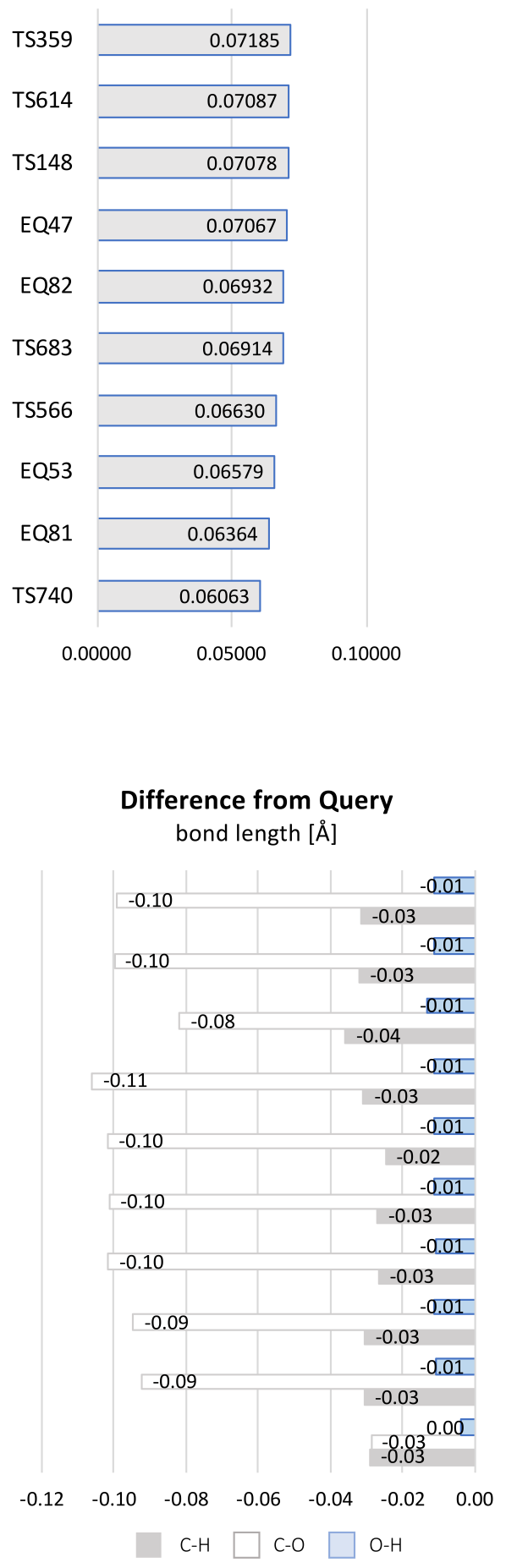

Difference from Query

torsion angle [degrees]

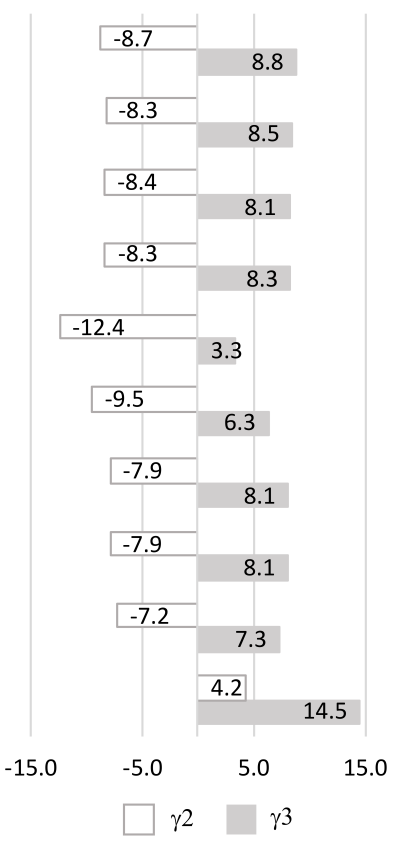

Difference from Query

bond angle [degrees]

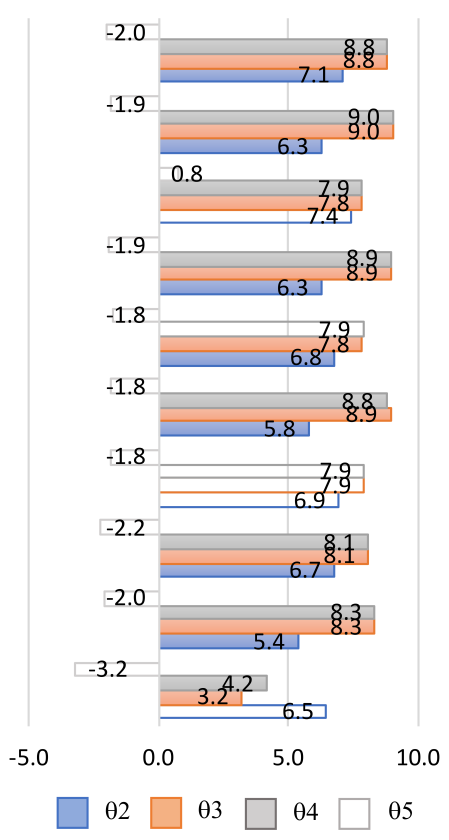

Figure 11: G-RMSD similarity indicator $\mu$ of structures with high similarity to query structure 4 and geometry differences. 
G-RMSD Similarity Index $\mu$
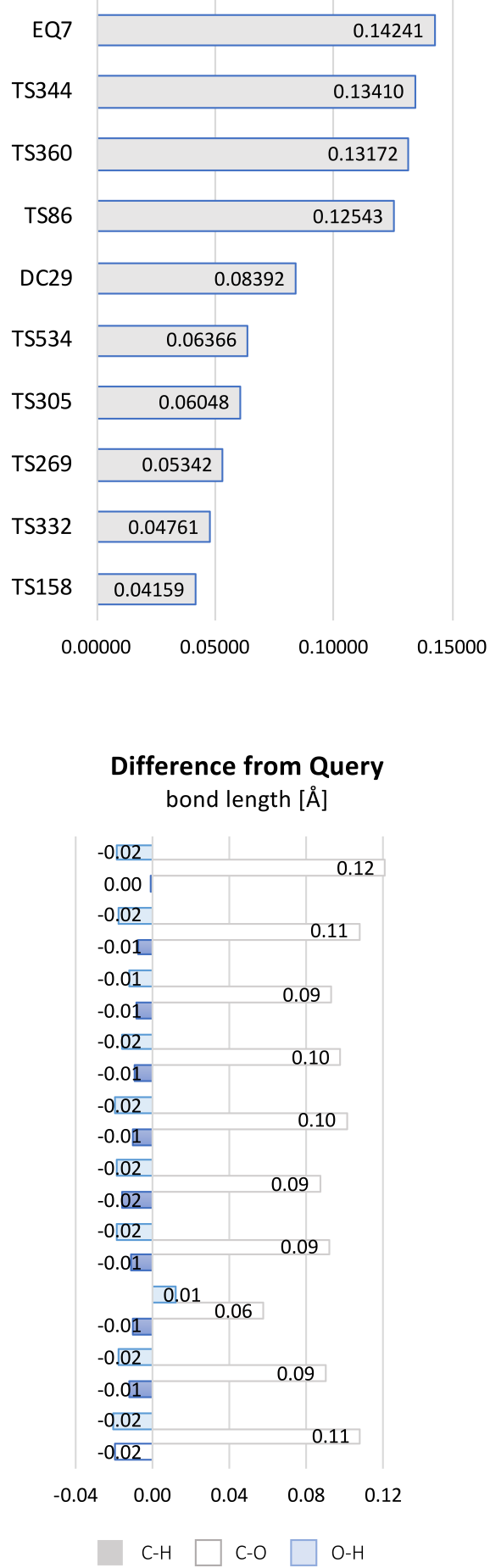

Difference from Query

torsion angle [degrees]

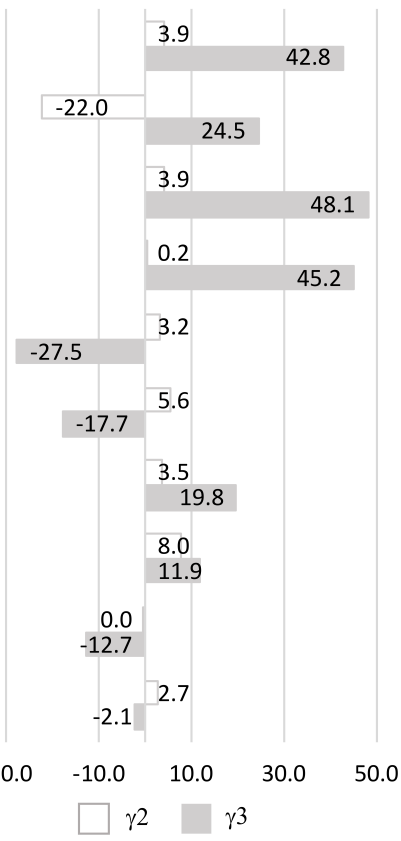

Difference from Query bond angle [degrees]

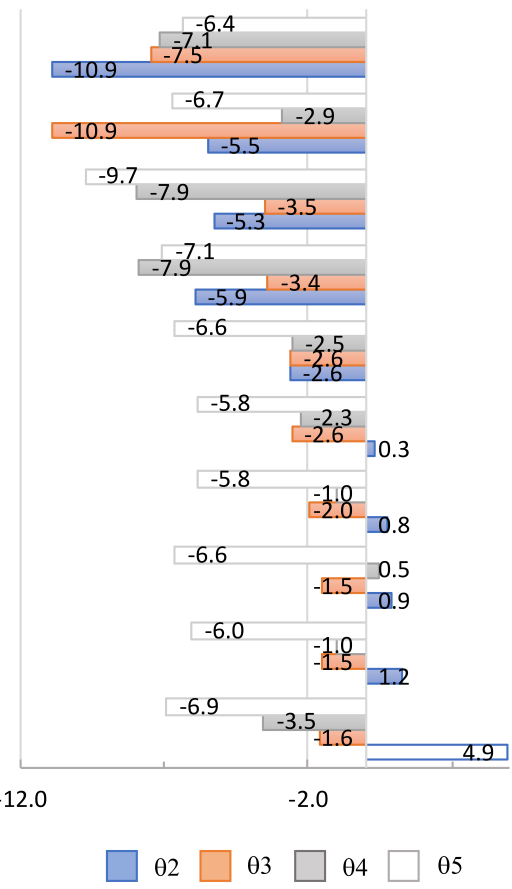

Figure 12: G-RMSD similarity indicator $\mu$ of structures with high similarity to query structure $\mathbf{5}$ and geometry differences. 
than 0.06 are in the peak at far left-hand side of the histogram of Figure 7, and the other five structures are in the highest peak in blue.

The difference of the dihedral angle $\gamma 1$ for query 3 is found to be in a wide range from 8.3 to 24.8 degrees in the top ten structures, and the $\mu$ values are also in a wide range from 0.03637 to 0.11041 (Figure 10). No large differences were found in the other geometry. The $\gamma 1$ is the out-of-plane angle of the methyl group. The methyl group of $\mathbf{3}$ is completely flat $(\gamma 1=0)$. Figure 10 shows the good correlation between $\mu$ and $\gamma$ values. The difference of $\gamma$ values represents that the $\mathrm{CH}_{3}$ partial structures of the top three (DC58, TS318, and TS319) are fairly flat, which can be seen also in Figure 8b.

There are no large differences in the geometry and the $\mu$ values for the top ten structures matched with query 4 (Figures $8 \mathrm{c}$ and 11). A good correlation can be found between the $\mu$ values and the sum of the absolute values of $\gamma 2$ and $\gamma 3(|\gamma 2|+|\gamma 3|)$, except for TS740. The highest similarity of TS740 to the query is assumed to be due to the high similarity in the $\mathrm{C}-\mathrm{O}$ bond length and the $\mathrm{H}-\mathrm{C}-\mathrm{O}$ angles, $\theta 2$ and $\theta 3$.

Query 5, a $\mathrm{CH}_{2} \mathrm{OH}$ cation, adopts a flat structure and the dihedral angles around the C-O bond, $\gamma 2$ and $\gamma 3$, are 0 degree (Figure 6). The structures with high similarity to the query also adopt a flat structure with cationic property (Figures $8 \mathrm{~d}$ and 12). The $\mu$ values to query $\mathbf{5}$ has a good correlation with the $|\gamma 2|+|\gamma 3|$ values.

These applications demonstrated that the G-RMSD method is useful also for 3D-partial structure search. The 3D-partial structure search successfully found molecules with similar 3D-partial structures from the dataset containing not only EQ but also TS and DC structures. All results show a good correlation between the $\mu$ values and the similarity in their 3Dgeometry. The G-RMSD method distinguished atom and bond types by only using the Cartesian coordinates of atoms, and the $\mu$ values sensitively reflect the similarity of different geometrical structures.

\section{CONCLUSIONS}

We introduced a new method to determine 3D-molecular similarity G-RMSD, build upon the RMSD method. G-RMSD is an optimization method to get an optimal superimposition 
for minimum RMSD without a unique atom mapping. It removed the restrictions of RMSD, which can only be used for comparing molecules of the same size and requires a unique atom mapping or a fixed superimposition between the molecules. G-RMSD is applicable to comparison not only between the same size of structures but also molecules of different size. The molecular similarity value $\mu$ that can be measured only from atomic Cartesian coordinates sensitively reflects the similarity of different geometrical structures including atom- and bond types.

The G-RMSD method is a well-defined algorithm, which makes a simple usage possible. It has this property in common with many useful mathematical methods developed for chemoinformatics. Because of its property the G-RMSD method can be applied in a wide variety of situations. All the applications shown in this article are to organic molecules, but it can also be applied to various types of structures, including inorganic molecules, biomolecules, polymers, crystal structures, and amorphous materials.

The burgeoning of data-driven chemistry, e.g. machine learning (ML) and artificial intelligence (AI) chemistry, has been expanding the chemical space with larger quantity and higher quality of data. QM-based chemical database with the structural hierarchy from simple topological to full 3D information including transition state and non-valence structures have been getting into the mainstream. We believe that the G-RMSD method will contribute to advanced studies handling such chemical information.

\section{Availability and requirements}

Program name: G-RMSD

Project home page: https://github.com/striwata/GRMSD

Programming language: MATLAB language

Requirements: MATLAB ${ }^{70}$

License: MIT license 


\section{Competing interests}

The authors declare that they have no competing interests.

\section{Authors' contributions}

H.S. designed the project and performed the QM calculations. S.I., T.F., and K.M. designed the algorithm. T.F. and K.M. programmed the algorithm and performed the G-RMSD calculations. T.F., K.M., S.I., and H.S. analyzed the results of the G-RMSD calculations and wrote the manuscript.

\section{Acknowledgments}

The authors thanks Prof. Koichi Ohno at Tohoku University and Institute for Quantum Chemical Exploration (IQCE) for the help of GRRM calculations for $\mathrm{C}_{2} \mathrm{H}_{4} \mathrm{O}_{2}$. H.S. thanks Prof. J,rg Hutter at University of Zurich for helpful discussions.

\section{Supporting Information}

Additional Supporting Information may be found in the online version of this article. 


\section{References}

1. R. Todeschini and V. Consonni, in Handbook of Molecular Descriptors (Wiley-VCH, Weinheim, 2000), pp. 395-403.

2. T. Engel, in Chemoinfomatics, A Textbook, edited by J. Gasteiger and T. Engel (WileyVCH, Weinheim, 2003), pp. 15-168.

3. N. Kochev, V. Monev, and I. Bangov, in Chemoinfomatics, A Textbook, edited by J. Gasteiger and T. Engel (Wiley-VCH, Weinheim, 2003), pp. 291-318.

4. P. Willet, WIREs Data Mining and Knowledge Discovery 1, 241 (2011).

5. The American Chemical Society, The Chemical Abstracts Service, https://www.cas.org, accessed 05 May 2019.

6. G. Schneider, W. Neidhart, T. Giller, and G. Schmid, Angewandte Chemie International Edition 38, 2894 (1999).

7. L. C. Blum and J.-L. Reymond, Journal of American Chemical Society 131, 8732 (2009).

8. J.-L. Reymond, L. Ruddigkeit, L. Blum, and R. van Deursen, WIREs Data Mining and Knowledge Discovery 2, 717 (2012).

9. L. Ruddigkeit, R. van Deursen, L. Blum, and J.-L. Reymond, Journal of Chemical Information and Modeling 52, 2864 (2012).

10. H. Satoh, H. Koshino, K. Funatsu, and T. Nakata, Journal of Chemical Information and Computer Science 40, 622 (2000).

11. H. Satoh, H. Koshino, K. Funatsu, and T. Nakata, Journal of Chemical Information and Computer Science 41, 1106 (2001).

12. H. Satoh, H. Koshino, and T. Nakata, Journal of Computer Aided Chemistry 3, 48 (2002).

13. H. Satoh, Croatica Chemica Acta 80, 217 (2007). 
14. S. De, A. P. Bartók, G. Csányi, and M. Ceriotti, Phys. Chem. Chem. Phys. 18, 13754 (2016).

15. S. De, F. Musil, C. Baldauf, and M. Ceriotti, Journal of Chemoinformatics p. 9:6 (2017).

16. RSCB, Rscb protein data bank, https://rcsb.org, accessed 05 May 2019.

17. H. M. Berman, J. Westbrook, Z. Feng, G. Gilliland, T. Bhat, H. Weissig, I. N. Shindyalov, and P. E. Bourne, Nucleic Acids Research 28, 235 (2000).

18. CSD, The Cambridge Structural Database, https://ccdc.cam.ac.uk, accessed 05 May 2019.

19. C. R. Groom, I. J. Bruno, M. P. Lightfoot, and S. C. Ward, Acta Crystallographica Section B B72, 171 (2016).

20. K. Hori, Journal of Computer Aided Chemistry 2, 37 (2001).

21. G. Montavon, M. Rupp, V. Gobre, A. Vazquez-Mayagoitia, K. Hansen, A. Tkatchenko, K.-R. Müller, and O. A. von Lilienfeld, New Journal of Physics 15, 095003 (2013), URL http: //stacks.iop.org/1367-2630/15/i=9/a=095003.

22. R. Ramakrishnan, P. O. Dral, M. Rupp, and O. A. von Lilienfeld, Scientific Data p. $1: 140022(2014)$.

23. R. Ramakrishnan, M. Hartmann, E. Tapavicza, and O. A. von Lilienfeld, The Journal of Chemical Physics 143, 084111 (2015).

24. M. Nakata, AIP Conference Proceedings 1702, 090058 (2015).

25. M. Nakata and T. Shimazaki, Journal of Chemical Information and Modeling 57, 1300 (2017).

26. M. Nakata, The PubChemQC Project, http://pubchemqc.riken.jp, accessed 05 May 2019.

27. H. Satoh, T. Oda, K. Nakakoji, T. Uno, S. Iwata, and K. Ohno, Journal of Computer Chemistry Japan 14, 77 (2015). 
28. H. Satoh, T. Oda, K. Nakakoji, T. Uno, S. Iwata, and K. Ohno, Reaction Map Database, http: //www.reactionroutemap. com/rmapserver (2014), accessed 05 May 2019.

29. H. Satoh, T. Oda, K. Nakakoji, T. Uno, S. Iwata, and K. Ohno, Reaction Map Database Server, https://github.com/ReactionMap/RMapServer (2018), accessed 05 May 2019.

30. F. Brockherde, L. Vogt, L. Li, M. E. Tuckerman, K. Burke, and K.-R. Müller, Nature Communications 8, 872 (2017).

31. C. R. Collins, G. J. Gordon, O. A. von Lilienfeld, and D. J. Yaron, The Journal of Chemical Physics 148, 241718 (2018).

32. J.-F. Gibrat, T. Modej, and S. H. Bryant, Current Opinion in Structural Biology 6, 377 (1996).

33. K. Kedem, L. P. Chew, and R. Elber, PROTEINS: Structure, Function, and Genetics 37, 554 (1999).

34. R. Bruüschweiler, PROTEINS: Structure, Function, and Genetics 50, 26 (2003).

35. M. Shatsky, A. Shulman-Peleg, R. Nussinov, and H. J. Wolfson, Journal of Computational Biology 13, 407 (2006).

36. T. Shibuya, J. Jansson, and K. Sadakane, Algorithms for Molecular Biology 5, 1 (2010).

37. S. C. Li, Algorithms for Molecular Biology 8 (2013).

38. T. Madej, C. J. Lanczycki, D. Zhang, P. A. Thiessen, R. C. Geer, A. Marchler-Bauer, and S. H. Bryant, Nucleic Acids Research pp. 297-303 (2014).

39. L. Zhu, M. Amsler, T. Fuhrer, B. Schaefer, S. Faraji, S. Rostami, S. A. Ghasemi, A. Sadeghi, M. Grauzinyte, C. Wolverton, et al., The Journal of Chemical Physics 144, 034203 (2016).

40. K. S. Arun, T. S. Huang, and S. D. Blostein, IEEE Transactions on Pattern Analysis and Machine Intelligence 9, 698 (1987). 
41. P. J. de Rezende and D. Lee, Algorithmica 13, 387 (1995).

42. K. Ohno and S. Maeda, Chemical Physics Letters 384, 277 (2004).

43. S. Maeda and K. Ohno, Chemistry Letters 33, 1372 (2004).

44. S. Maeda and K. Ohno, Chemical Physics Letters 398, 240 (2004).

45. S. Maeda and K. Ohno, Chemical Physics Letters 404, 95 (2005).

46. S. Maeda, Y. Harabuchi, Y. Osada, T. Taketsugu, K. Morokuma, and K. Ohno, GRRM14, https://iqce.jp/GRRM/index_e.shtml (2015), accessed 05 May 2019.

47. T. Nukada, A. Bérces, and D. M. Whitfield, Carbohydrate Research 337, 765 (2002).

48. H. Satoh, H. S. Hansen, S. Manabe, W. F. van Gunsteren, and P. H. Hünenberger, Journal of Chemical Theory and Computations 6, 1783 (2010).

49. A. Ardèvol and C. Rovira, Angewandte Chemie, International Edition 50, 10897 (2011).

50. D. M. Whitfield, Carbohydrate Research 356, 180 (2012).

51. H. Satoh and S. Manabe, Chemical Society Reviews 42, 4297 (2013).

52. H. Satoh and T. Nukada, Trends in Glycoscience and Glycotechnology pp. 11-27 (2014).

53. H. B. Mayes, L. J. Broadbelt, and G. T. Beckham, Journal of the American Chemical Society 136, 1008 (2014).

54. L. Bohé and D. Crich, Carbohydrate Research 403, 48 (2015).

55. D. M. Whitfield, Carbohydrate Research 403, 69 (2015).

56. H. Satoh, T. Oda, K. Nakakoji, T. Uno, H. Tanaka, S. Iwata, and K. Ohno, Journal of Chemical Theory and Computation 12, 5293 (2016).

57. IUPAC-IUB Joint Commission on Biochemical Nomenclature (JCBN), European Journal of Biochemistry 111, 295 (1980). 
58. IUPAC-IUB Joint Commission on Biochemical Nomenclature (JCBN), Archives of Biochemistry and Biophysics 207, 469 (1981).

59. IUPAC-IUB Joint Commission on Biochemical Nomenclature (JCBN), Pure and Applied Chemistry 53, 1901 (1981).

60. D. Cremer and J. Pople, Journal of the American Chemical Society 97, 1354 (1975).

61. D. Cremer, Journal of Physical Chemistry 94, 5502 (1990).

62. A. Bérces, D. M. Whitfield, and T. Nukada, Tetrahedron 57, 477 (2001).

63. A. Bérces, D. M. Whitfield, and T. Nukada, Characterization of Six-membered Ring Conformations, http://6ring.bio.nrc.ca (2001), accessed 05 May 2019.

64. RSCB, The Open Graph Viz Platform, https://gephi.org, accessed 05 May 2019.

65. H. Tokoyama, H. Yamakado, S. Maeda, and K. Ohno, Bulletin of the Chemical Society of Japan 88, 1284 (2015).

66. T. Tokiwa, N. Kishimoto, and K. Ohno, Chemical Physics Letters 641, 97 (2015).

67. K. Ohno, N. Kishimoto, T. Iwamoto, and H. Satoh, Journal of Computational Chemistry 38, 669 (2017).

68. H. Satoh, T. Oda, K. Nakakoji, T. Uno, S. Iwata, and K. Ohno, RMapViewer, https://github.com/ReactionMap/RMapViewer (2014), accessed 05 May 2019.

69. M. J. Frisch, G. W. Trucks, H. B. Schlegel, G. E. Scuseria, M. A. Robb, J. R. Cheeseman, G. Scalmani, V. Barone, G. A. Petersson, H. Nakatsuji, et al., Gaussian 16 Revision B.01 (2016), gaussian Inc. Wallingford CT.

70. MathWorks, MATLAB, https://www.mathworks.com, accessed 05 May 2019. 


\title{
Supporting Information to:
}

\section{G-RMSD: Root Mean Square Deviation Based Method for Three- dimensional Molecular Similarity Determination}

\author{
Tomonori Fukutani, ${ }^{1}$ Kohei Miyazawa, ${ }^{1}$ Satoru Iwata, ${ }^{1 *}$ and Hiroko Satoh ${ }^{2,3^{*}}$ \\ 1. Department of Mathematical Informatics, The University of Tokyo, Hongo 7-3-1, Bunkyo, 113-8654, Tokyo, Japan \\ 2. Department of Chemistry, University of Zurich, Winterthrerstrasse 190, 8057 Zurich, Switzerland \\ 3. Research Organization of Information and Systems (ROIS), Toranomon 4-3-13, Minato, 105-0001, Tokyo, Japan
}

\section{S1. Standard RMSD and G-RMSD}

\section{S1.1. Difference between standard RMSD and G-RMSD}

The difference between the standard RMSD and G-RMSD methods are summarized in Table S11. The standard RMSD value between molecules $A$ and $B$ is calculated with unique atom mapping by Eq.(1) It is called here standard RMSD-1. The standard RMSD-1 calculations need unique atom mapping together with Cartesian coordinates. In order to calculate RMSD values without unique atom mapping, permutation $\sigma(i)$ has to be added (Eq.(3)). It is called here standard RMSD-2. It is known that Eq.(3), the equation without unique atom mapping, can be solved with Hungarian method, if the rotation $R$ and translation $v$ are fixed, meaning, with a fixed superimposition.

G-RMSD is an efficient optimization method that can calculate minimum RMSD against all the three variables: $R$ (rotation), $v$ (translation), and, $\sigma$ (permutation). This means, G-RMSD needs neither unique atom mapping nor a fixed superimposition. G-RMSD needs only Cartesian coordinates, whereas the standard RMSD- 1 and 2 cannot be calculated only from Cartesian coordinates.

Table S1-1. Comparison between standard RMSD and G-RMSD.

\begin{tabular}{|c|c|c|c|}
\hline & Standard RMSD-1 & Standard RMSD-2 & G-RMSD \\
\hline Target value & $\mu(A, B)$ (minimum $R M S D$ ) & $\mu(A, B)$ (minimum RMSD) & $\mu(A, B)$ (minimum RMSD) \\
\hline $\begin{array}{l}\text { Equations and variables } \\
\text { to calculate } \mu(A, B)\end{array}$ & $\begin{array}{l}\mathrm{Eq}(1) \\
\text { RMSD is minimized } \\
\text { against } a, b, R \text {, and } v .\end{array}$ & $\begin{array}{l}\text { Eq (3) } \\
\text { RMSD is minimized } \\
\text { against for } a, b \text {, and } \sigma . \\
R \text { and } v \text { are fixed. }\end{array}$ & $\begin{array}{l}\mathrm{Eq}(3) \\
\mathrm{RMSD} \text { is minimized } \\
\text { against } a, b, \sigma, R \text {, and } v \text {. }\end{array}$ \\
\hline $\begin{array}{l}\text { What information is } \\
\text { needed to run }\end{array}$ & $\begin{array}{l}\text { (1) Cartesian coordinates } \\
\text { (2) Unique atom } \\
\text { mapping }\end{array}$ & $\begin{array}{l}\text { (1) Cartesian coordinates } \\
\text { (2) Fixed superimposition }\end{array}$ & (1) Cartesian coordinates \\
\hline $\begin{array}{l}\text { What is done in } \\
\text { optimization }\end{array}$ & $\begin{array}{l}\text { RMSD is minimized by } \\
\text { rotation (changing R) } \\
\text { and translation } \\
\text { (changing } v \text { ) of the } \\
\text { position of molecules } \\
\text { A/B. }\end{array}$ & $\begin{array}{l}\text { RMSD is minimized by } \\
\text { changing atom-pair } \\
\text { mapping with } \\
\text { permutation } \sigma \text {. } \\
\text { The position of molecules } \\
\text { A and B is fixed. }\end{array}$ & $\begin{array}{l}\text { RMSD is minimized by } \\
\text { rotation } R \text {, translation } v \text {, } \\
\text { and changing atom-pair } \\
\text { mapping } \sigma \text {. }\end{array}$ \\
\hline $\begin{array}{l}\text { What user has to do } \\
\text { before calculations }\end{array}$ & $\begin{array}{l}\text { Specify unique atom } \\
\text { mapping between } \\
\text { molecules } A \text { and } B\end{array}$ & $\begin{array}{l}\text { Superimpose molecules } A \\
\text { and } B\end{array}$ & Nothing \\
\hline 3D-DB search & impossible & impossible & possible \\
\hline
\end{tabular}




\section{S1.2. 3D-Similarity Measurement of Six-membered Rings of $\alpha$-D-Glucose}

The difference between standard RMSD-1 and G-RMSD in measuring the 3D-similarity of ${ }^{4} C_{1}$ and ${ }^{1} C_{4}$ conformers of $\alpha$-D-glucose is illustrated. If focusing only on the six-membered rings, the ${ }^{4} C_{1}$ and ${ }^{1} C_{4}$ conformers are in principle the same 3 D-geometry including the atom types (Figure S1-1). The difference of the orientation of substituents (e.g., axial or equatorial hydroxyl groups) can make the bond lengths and angles slightly changed. Therefore, the correct answer of 3D-similarity measurement between them is the same or very similar.

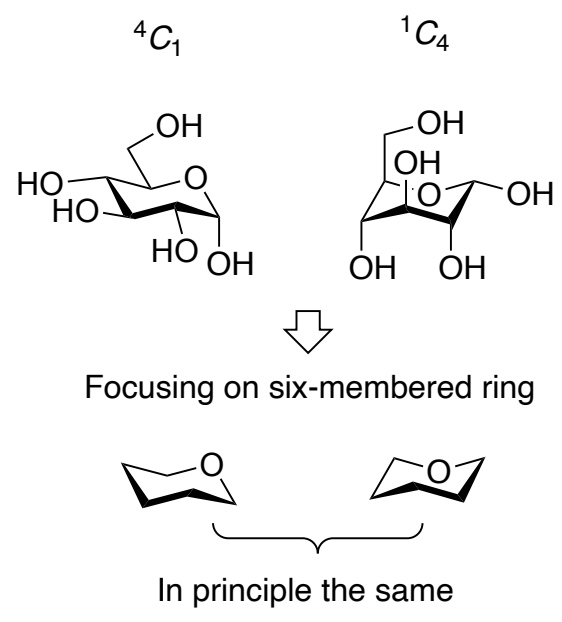

Figure S1-1. Correct Answer of 3D-Similarity Measurement between ${ }^{4} C_{1}$ and ${ }^{1} C_{4}$ Six-membered Rings of $\alpha$-D-Glucose. If focusing only on six-membered ring of ${ }^{4} C_{1}$ and ${ }^{1} C_{4}$, they are in principle the same.

Figure S1-2 shows the difference between standard RMSD-1 and G-RMSD in the 3D-similarity measurement for the two rings. Standard RMSD-1 needs unique atom mapping between the two rings. Optimization is done to obtain the minimum RMSD $\mu(\mathrm{A}, \mathrm{B})$ by rotating and translating the two ring position as keeping the atom mapping. As shown in Figure S1-2, standard RMSD-1 cannot achieve the correct superimposition due to the restriction of the unique atom mapping. G-RMSD does not need the unique atom mapping as prerequisite information. Optimization is done to obtain the minimum RMSD $\mu(\mathrm{A}, \mathrm{B})$ by rotating and translating the two ring position. The atom-pair mapping is also changed. As shown in Figure S1-2, G-RMSD can achieve the correct superimposition. Incidentally, standard RMSD2 cannot be applied to this measurement because of the restriction of the prerequisite superimposition.

\section{Standard RMSD-1}

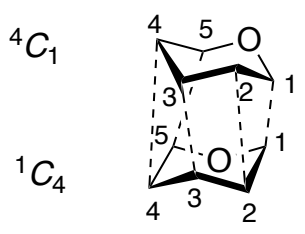

${ }^{4} C_{1}$ and ${ }^{1} C_{4}$ rings cannot be correctly superimposed due to the restriction of unique atom mapping.

\section{G-RMSD}

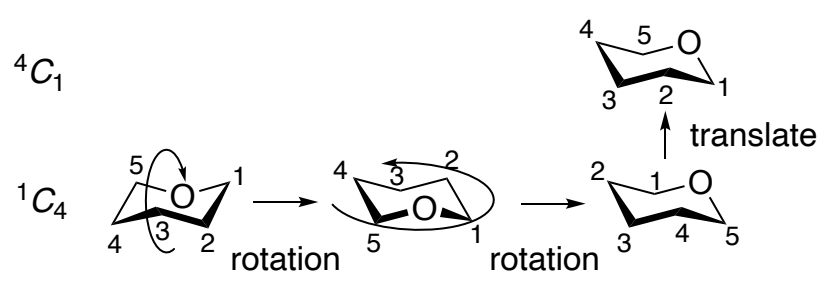

${ }^{4} C_{1}$ and ${ }^{1} C_{4}$ rings are correctly superimposed by rotation, translation, and considering the permutation of atom-pair mapping.

Figure S1-2. Comparison between Standard RMSD-1 and G-RMSD in 3D-Similarity Measuring ${ }^{4} C_{1}$ and ${ }^{1} C_{4}$ Six-membered Rings. Standard RMSD-1 cannot match the two same conformations due to the restriction of the unique atom mapping. G-RMSD can match them by rotation, translation, and considering the permutation of atom-pair mapping. 